\title{
NEWTON POLYHEDRA AND ORDER OF CONTACT ON REAL HYPERSURFACES
}

\author{
JOE KAMIMOTO
}

\begin{abstract}
The purpose of this paper is to investigate order of contact on real hypersurfaces in $\mathbb{C}^{n}$ by using Newton polyhedra which are important notion in the study of singularity theory. To be more precise, an equivalence condition for the equality of regular type and singular type is given by using the Newton polyhedron of a defining function for the respective hypersurface. Furthermore, a sufficient condition for this condition, which is more useful, is also given. This sufficient condition is satisfied by many earlier known cases (convex domains, pseudoconvex Reinhardt domains and pseudoconvex domains whose regular types are 4, etc.). Under the above conditions, the values of the types can be directly seen in a simple geometrical information from the Newton polyhedron.
\end{abstract}

\section{Contents}

1. Introduction

2. Elementary properties of Newton polyhedra

3. Remarks on nondegeneracy condition

4. A new invariant and adapted coordinates

5. Proof of Theorem 1.1

6. Order of vanishing

7. Asymptotics of $F \circ \gamma$

8. Order of contact for smooth functions

9. Proof of Theorem 1.3

10. On normalized coordinates 27

11. Pseudoconvexity 30

12. The semiregular ( $h$-extendible) case 32

13. The case of Reinhardt domains 34

14. The case where the regular type is $4 \quad 36$

15. The case of star-shaped domains 38

References 39

2010 Mathematics Subject Classification. 32F18.

Key words and phrases. Singular type, Regular type, Finite type, Order of contact, Newton polyhedra, Nondegeneracy condition. 


\section{INTRODUCTION}

Let $M$ be a $\left(C^{\infty}\right)$ real hypersurface in $\mathbb{C}^{n}$ and let $p$ lie on $M$. Let $r$ be a local defining function for $M$ near $p(\nabla r \neq 0$ when $r=0)$. D'Angelo [7], 8] defined an important invariant $\Delta_{1}(M, p)$, the singular type of $M$ at $p$, by

$$
\Delta_{1}(M, p):=\sup _{\gamma \in \Gamma} \frac{\operatorname{ord}(r \circ \gamma)}{\operatorname{ord}(\gamma-p)}
$$

and established its fundamental properties. Here $\Gamma$ denotes the set of (germs of) holomorphic mappings $\gamma:(\mathbb{C}, 0) \rightarrow\left(\mathbb{C}^{n}, p\right)$ with $\gamma \not \equiv p$. For a $C^{\infty}$ mapping $h: \mathbb{C} \rightarrow \mathbb{C}$ or $\mathbb{C}^{n}$ such that $h(0)=0$, let ord $(h)$ denote the order of vanishing of $h$ at 0 (see Section 6 for its exact definition).

As is well-known, the invariant (1.1) plays important roles in the study of the $\bar{\partial}$ Neumann problem. Let $M$ be the boundary of a smoothly bounded pseudoconvex domain in $\mathbb{C}^{n}$. It was shown by Catlin [5], 6] that the finite type condition at $p$ (i.e., $\left.\Delta_{1}(M, p)<\infty\right)$ is equivalent to the condition that the subelliptic estimate holds near $p$. Furthermore, since this invariant is deeply connected with many analytical subjects in the study of several complex variables, its properties have been investigated from various points of view.

Computing the singular type (1.1), or even determining whether the respective type is finite, is not always simple matter. This difficulty is often caused by the definition in which all the mapping in $\Gamma$ must be treated. Thus, it is desirable that $\Gamma$ is replaced by an appropriate smaller class which is easily treated. It has been known in special cases, below, that in order to decide the type, it suffices to check all the regular holomorphic mappings in $\Gamma$. To be more specific, let us explain this issue. The regular type of $M$ at $p$ is defined by

$$
\Delta_{1}^{\mathrm{reg}}(M, p):=\sup _{\gamma \in \Gamma_{\mathrm{reg}}}\{\operatorname{ord}(r \circ \gamma)\},
$$

where $\Gamma_{\text {reg }}$ denotes the set of holomorphic mappings $\gamma:(\mathbb{C}, 0) \rightarrow\left(\mathbb{C}^{n}, p\right)$ with ord $(\gamma)=$ 1 (i.e. $\nabla \gamma(0) \neq 0$ ). Since $\Gamma_{\text {reg }} \subset \Gamma$, the inequality $\Delta_{1}(M, p) \geq \Delta_{1}^{\text {reg }}(M, p)$ always holds. We will consider the following question.

Question 1. When does the following equality hold?

$$
\Delta_{1}(M, p)=\Delta_{1}^{\mathrm{reg}}(M, p) .
$$

Until now, there have been results showing that the equality (1.3) holds for $M$, where $M$ is the smooth boundary of the following domains:

(A) Convex domains (McNeal [30]);

(B) Star-shaped domains (Boas-Straube [4]);

(C) Pseudoconvex Reinhardt domains (Fu-Isaev-Krantz [15]);

(D) Pseudoconvex (or property PS) domains with $\Delta_{1}^{\text {reg }}(M, p)=4$ (McNeal-Mernik [31], D'Angelo [9]). 
The methods used in the above cited papers are so different that it seems difficult to find common essential characteristics in them to establish the equality (1.3). In this paper, we investigate conditions for the equality (1.3) by using the Newton polyhedron of a defining function for $M$, which is an important notion in the study of singularity theory (c.f. [1], [33]), and interpret this phenomenon containing the above cases based on the geometry of the respective Newton polyhedron.

Let us define the Newton polyhedron of a smooth function $F$ defined near the origin in $\mathbb{C}^{n}$. The Taylor series expansion of $F$ at the origin is

$$
F(z, \bar{z}) \sim \sum_{\alpha, \beta \in \mathbb{N}_{0}^{n}} C_{\alpha \beta} z^{\alpha} \bar{z}^{\beta} \quad \text { with } C_{\alpha \beta}=\frac{1}{\alpha ! \beta !} D^{\alpha} \bar{D}^{\beta} F(0,0) .
$$

The support of $F$ is defined by $S(F)=\left\{\alpha+\beta \in \mathbb{N}_{0}^{n}: C_{\alpha \beta} \neq 0\right\}$. The Newton polyhedron of $F$ is defined by

$$
\mathcal{N}_{+}(F)=\text { The convex hull of }\left(\bigcup_{\alpha+\beta \in S(F)}\left(\alpha+\beta+\mathbb{R}_{\geq}^{n}\right)\right) .
$$

The Newton diagram $\mathcal{N}(F)$ of $F$ is defined to be the union of the bounded faces of $\mathcal{N}_{+}(F)$. We use coordinates $(\xi)=\left(\xi_{1}, \ldots, \xi_{n}\right)$ for points in the plane containing the Newton polyhedron. The following classes of functions $F$ simply characterized by using their Newton polyhedra often appear in this paper:

- $F$ is called to be flat if $\mathcal{N}_{+}(F)$ is an empty set.

- $F$ is called to be convenient if $\mathcal{N}_{+}(F)$ meets every coordinate axis.

Let $(z)=\left(z_{1}, \ldots, z_{n}\right)$ be a holomorphic coordinate around $p$ such that $p=0$. Let $r$ be a local defining function for $M$ near $p$ on the coordinate $(z)$. For a given tuple $(M, p ;(z))$, we define a quantity $\rho_{1}(M, p ;(z)) \in \mathbb{N} \cup\{\infty\}$ as follows. If $r$ is convenient, then let

$$
\rho_{1}(M, p ;(z)):=\max \left\{\rho_{j}(r): j=1, \ldots, n\right\},
$$

where $\rho_{j}(r)$ be the coordinate of the point at which the Newton diagram $\mathcal{N}(r)$ intersects the $\xi_{j}$-axis (see Section 2.3). Otherwise, let $\rho_{1}(M, p ;(z)):=\infty$. We remark that $\rho_{1}(M, p ;(z))$ depends on the chosen coordinate $(z)$, but it is independent of the choice of defining functions after fixing a coordinate (see Section 2.4).

Now, let us give an equivalence condition for the equality (1.3) by using the quantity $\rho_{1}(M, p ;(z))$.

Theorem 1.1. When $\Delta_{1}^{\mathrm{reg}}(M, p)<\infty$, the following two conditions are equivalent.

(i) $\Delta_{1}(M, p)=\Delta_{1}^{\mathrm{reg}}(M, p)$;

(ii) There exists a holomorphic coordinate $(z)$ at $p$ such that $\Delta_{1}(M, p)=\rho_{1}(M, p ;(z))$.

Of course, when $\Delta_{1}^{\mathrm{reg}}(M, p)=\infty$, the equality (1.3) holds. The proof of Theorem 1.1 will be given in Section 5 . 
It seems to be not so easy to see what kinds of coordinates satisfy the condition in (ii) in Theorem 1.1. In order to consider a useful class of coordinates, let us introduce a concept "nondegeneracy condition" on a smooth function $F$ defined near the origin in $\mathbb{C}^{n}$.

Let $\kappa$ be a bounded face of $\mathcal{N}_{+}(F)$. The $\kappa$-part of $F$ is the polynomial defined by

$$
F_{\kappa}(z, \bar{z})=\sum_{\alpha+\beta \in \kappa} C_{\alpha \beta} z^{\alpha} \bar{z}^{\beta}
$$

The set of complex curves $\widetilde{\Gamma}_{\kappa}^{*}$ is defined by

$$
\widetilde{\Gamma}_{\kappa}^{*}:=\left\{\left(c_{1} t^{a_{1}}, \ldots, c_{n} t^{a_{n}}\right): c \in\left(\mathbb{C}^{*}\right)^{n}, t \in \mathbb{C}, a \in \mathbb{N}^{n} \text { determines } \kappa\right\},
$$

where $c=\left(c_{1}, \ldots, c_{n}\right) \in\left(\mathbb{C}^{*}\right)^{n}, a=\left(a_{1}, \ldots, a_{n}\right) \in \mathbb{N}^{n}$ and " $a \in \mathbb{N}^{n}$ determines $\kappa$ " means that the equality $\left\{\xi \in \mathcal{N}_{+}(F):\langle a, \xi\rangle=l\right\}=\kappa$ holds for some $l \in \mathbb{N}$ (see Section 2.2).

Definition 1.2. Let $\kappa$ be a bounded face of $\mathcal{N}_{+}(F)$. The $\kappa$-part $F_{\kappa}$ of $F$ is said to be nondegenerate if

$$
F_{\kappa} \circ \gamma \not \equiv 0 \quad \text { for any } \gamma \in \widetilde{\Gamma}_{\kappa}^{*} \text {. }
$$

A function $F$ is said to be nondegenerate, if $F_{\kappa}$ is nondegenerate for every bounded face $\kappa$ of $\mathcal{N}_{+}(F)$.

Detailed properties of the above condition will be explained in Section 3. This condition is analogous one introduced by Kouchnirenko [27] (see Section 3.1 (5)). His nondegeneracy condition often plays important roles in resolution of singularities by using the geometry of Newton polyhedra. The nondegeneracy condition in Definition 1.2 is also essentially important in our analysis. Indeed, the following theorem shows that this condition gives sufficiently appropriate properties for the condition (ii) in Theorem 1.1.

Theorem 1.3. If there exists a holomorphic coordinate $(z)$ at $p$ such that $p=0$ on which a local defining function $r$ for $M$ is nondegenerate, then the following equalities holds:

$$
\Delta_{1}(M, p)=\Delta_{1}^{\mathrm{reg}}(M, p)=\rho_{1}(M, p ;(z)) .
$$

The above coordinate $(z)$ is said to be canonical for $M$ at $p$. The proof of Theorem 1.3 will be given in Section 9 below.

From Theorem 1.3, when the Newton polyhedron $\mathcal{N}_{+}(r)$ can be explicitly described on a canonical coordinate, the exact values of the regular and singular types (including the case of $\infty)$ can be directly seen, which are equal to $\rho_{1}(M, p ;(z))$. In particular, the convenience condition (i.e. $\mathcal{N}_{+}(r)$ meets every coordinate axis) determines whether the type of $p$ is finite. 
Although many important properties of the regular and singular types can be understood on canonical coordinates, it is another serious issue to determine whether canonical coordinates exist for a given real hypersurface and, if they exist, to actually construct these coordinates. We consider this issue not only in the above mentioned cases (A), (C), (D) but also in more general cases.

Theorem 1.4. If $M$ and $p \in M$ satisfy one of the following conditions:

(i) $M$ is of semiregular type (h-extendible) at $p$ (see Lemma 12.2);

(ii) $M$ is the boundary of a pseudoconvex Reinhardt domain ( $p$ is any point);

(iii) $M$ satisfies property $\mathbf{P S}$ at $p$ with $\Delta_{1}^{\mathrm{reg}}(M, p)=4$,

then $M$ admits canonical coordinates at $p$.

The cases (i), (ii), (iii) in Theorem 1.4 are respectively treated in Theorems 12.3, 13.4, 14.1, below, whose proofs will be given after their statements. More precise investigation is seen in Sections 12-14. It follows from Theorem 1.4 that Theorem 1.3 includes the above mentioned results on (A), (C), (D).

Remark 1.5. (1) There exist real hypersurfaces not admitting canonical coordinates. For example, it is easy to see that there is no canonical coordinates near the origin for the real hypersurfaces $M_{1}, M_{2}$ in $\mathbb{C}^{3}$, which are respectively defined by

$$
\begin{aligned}
& r_{1}(z, \bar{z})=2 \operatorname{Re}\left(z_{3}\right)+\left|z_{1}^{3}-z_{2}^{2}\right|^{2}, \\
& r_{2}(z, \bar{z})=2 \operatorname{Re}\left(z_{3}\right)+\left|z_{1}\right|^{2}\left|z_{2}\right|^{2}\left|z_{1}-z_{2}\right|^{2}+\left|z_{1}\right|^{10}+\left|z_{2}\right|^{10} .
\end{aligned}
$$

The functions $r_{1}, r_{2}$ are plurisubharmonic functions. Note that $r_{1}$ often appears in the studies of D'Angelo ([7], [8], etc.) and that $M_{2}$ belongs to the star-shaped case (B) ([4], see Section 15). It is easy to check the following:

$$
\begin{array}{ll}
\Delta_{1}\left(M_{1}, 0\right)=\infty, & \Delta_{1}^{\text {reg }}\left(M_{1}, 0\right)=6 ; \\
\Delta_{1}\left(M_{2}, 0\right)=10, & \Delta_{1}^{\text {reg }}\left(M_{2}, 0\right)=10 .
\end{array}
$$

It follows from (1.11) that the existence of canonical coordinates is not necessary for the equality of regular and singular types.

(2) Owing to Theorem 1.3, we can easily produce many examples of pseudoconvex hypersurfaces satisfying the equality (1.3), which are not contained in the cases in Theorem 1.4. For example, it will be easily recognized after understanding the investigation in Sections 12-14 that the pseudoconvex hypersurface defined by

$$
\operatorname{Re}(w)+\left|z_{1}\right|^{8}+\frac{15}{7}\left|z_{1}\right|^{2}\left(\operatorname{Re}\left(z_{1}\right)\right)^{6}+\left|z_{1} z_{2}\right|^{2}+\left|z_{2}\right|^{6}=0
$$

is not contained in any case in Theorem 1.4, but it admits a canonical coordinate at the origin and its regular and singular types of the origin are 8. Moreover, this example does not satisfy the hypothesis in the result (see Theorem 15.1) in [4]. 
Let us explain ideas of our analysis roughly. The substantial analysis of the types is to investigate the following order. Let $F$ be a smooth function defined near the origin in $\mathbb{C}^{n}$ with $F(0)=0$. The order of contact of $\gamma$ with $F$ is defined by

$$
O(F, \gamma):=\frac{\operatorname{ord}(F \circ \gamma)}{\operatorname{ord}(\gamma)} \quad \text { for } \gamma \in \Gamma
$$

where $\Gamma$ is the same as it in (1.1) with $p=0$. In order to understand the situation of the contact of $\gamma$ with $F$ in (1.12), we use the geometry of the Newton polyhedron of $F$ and the vector $\phi(\gamma)$ naturally defined by the order of vanishing of $\gamma$. Roughly speaking, the "distance" of the Newton polyhedron of $F$ from the origin expresses the "flatness" at the origin of the hypersurface $M$ defined by $F=0$. When the Newton polyhedron of $F$ becomes far from the origin, the flatness of $M$ becomes strong. Our issue about (1.12) is more complicated. The situation of flatness of the restriction of $M$ to the complex curve defined by $\gamma$ is investigated. We introduce a new quantity "distance of the Newton polyhedron of $F$ in the direction $\phi(\gamma)$ " and show that the order of contact (1.12) equals this distance under the nondegeneracy condition on $F$ in Definition 1.2. Furthermore, this distance can be clearly expressed by more simple geometrical information from the Newton polyhedron of $F$. Applying this expression to the computation of the two types in (1.1), (1.2), we can see that, under the nondegeneracy condition of a defining function, the regular and singular types agree and that they can be expressed by using a simple geometrical information from the Newton polyhedron.

The technique of using Newton polyhedra has many significant applications in singularity theory (c.f. [1], [33]). In particular, this technique has been great success in the study of the Łojasiewicz exponent (28, 16, 35, 34, etc.). It is known in 29 that the Łojasiewicz exponent of $F$ can be expressed in a similar form to (1.1). Roughly speaking, this exponent can be written as the supremum of the form (1.12) by replaced $F$ by $\nabla F$ where $F$ is holomorphic. Our study about the types is analogous to the above cited works on the Łojasiewicz exponent. There are also many interesting applications of Newton polyhedra to the other analytical subjects. We only refer for studies about the oscillatory integrals to [40], 36], [19], [23], etc. and for those about the Bergman kernel to [21], 11], [10], etc. As for study about order of contact approached from the singularity theory, there are important works due to McNeal-Némethi [32], G. Heier [18] and Fornæss and Stensønes [14. This paper mainly treats Question 1 but we believe that the Newton polyhedron technique may be applied to the other deep problems around the types.

This paper is organized as follows. In Sections 2-3, detailed properties and subtle remarks about the Newton polyhedron and the nondegeneracy condition are explained. In Section 4, we introduce a new invariant from the quantity (1.5) and show that this invariant equals the regular type in (1.2). Theorem 1.1 is proved in Section 5. Sections 6-8 are the most important parts in our analysis, which show that the order $O(F, \gamma)$ in (1.4) can be expressed by using the geometry of the Newton polyhedron of $F$ under 
the nondegeneracy condition. By using results obtained in Section 8, Theorem 1.3 can be easily shown in Section 9. In Sections 10-14, special cases satisfying the hypothesis in Theorem 1.3 are precisely investigated and, as a result, Theorem 1.4 is proved. In Section 15, the result on the star-shaped case in (B) due to Boas and Straube [4], which generalizes the result [30] and is not included in Theorem 1.3, is discussed.

In [22], part of results of this paper has been announced.

Notation, symbols and terminology.

- The following symbols are used:

$$
\begin{aligned}
& \mathbb{N}_{0}:=\{0\} \cup \mathbb{N}, \mathbb{R}_{>}:=\{x \in \mathbb{R}: x>0\} \\
& \mathbb{R}_{\geq}:=\{x \in \mathbb{R}: x \geq 0\}, \mathbb{C}^{*}:=\mathbb{C} \backslash\{0\}
\end{aligned}
$$

- Some specific vectors are denoted as follows.

$$
\begin{aligned}
& 0:=(0, \ldots, 0), \mathbf{1}:=(1, \ldots, 1), \infty:=(\infty, \ldots, \infty), \\
& \mathbf{e}_{j}:=(0, \ldots, \stackrel{(j)}{1}, \ldots, 0) \text { for } j=1, \ldots, n
\end{aligned}
$$

- The multi-indices are used as follows. For $z=\left(z_{1}, \ldots, z_{n}\right), \bar{z}=\left(\bar{z}_{1}, \ldots, \bar{z}_{n}\right), \in$ $\mathbb{C}^{n}, \alpha=\left(\alpha_{1}, \ldots, \alpha_{n}\right), \beta=\left(\beta_{1}, \ldots, \beta_{n}\right) \in \mathbb{N}_{0}^{n}$, define

$$
\begin{aligned}
& z^{\alpha}:=z_{1}^{\alpha_{1}} \cdots z_{n}^{\alpha_{n}}, \bar{z}^{\beta}:=\bar{z}_{1}^{\beta_{1}} \ldots \bar{z}_{n}^{\beta_{n}}, \\
& |\alpha|:=\alpha_{1}+\cdots+\alpha_{n}, \quad \alpha !:=\alpha_{1} ! \cdots \alpha_{n} !, \quad 0 !:=1, \\
& D^{\alpha}:=\frac{\partial^{|\alpha|}}{\partial z_{1}^{\alpha_{1}} \cdots \partial z_{n}^{\alpha_{n}}}, \quad \bar{D}^{\beta}:=\frac{\partial^{|\beta|}}{\partial \bar{z}_{1}^{\beta_{1}} \cdots \partial \bar{z}_{n}^{\beta_{n}}} .
\end{aligned}
$$

- For $\xi=\left(\xi_{1}, \ldots, \xi_{n}\right), \zeta=\left(\zeta_{1}, \ldots, \zeta_{n}\right) \in \mathbb{R}^{n}$, we denote $\langle\xi, \zeta\rangle=\sum_{j=1}^{n} \xi_{j} \zeta_{j}$.

- In this paper, we always consider smooth functions, mappings, real hypersurfaces and complex curves as their respective germs without any mentioning. The following rings of germs of $\mathbb{C}$-valued functions are considered:

$-C_{0}^{\infty}\left(\mathbb{C}^{n}\right)$ is the ring of germs of $C^{\infty}$ functions at the origin in $\mathbb{C}^{n}$.

$-\mathcal{O}_{0}(\mathbb{C})$ is the ring of germs of holomorphic functions at the origin in $\mathbb{C}$.

- We use coordinates $(\xi)=\left(\xi_{1}, \ldots, \xi_{n}\right)$ for points in the plane containing the Newton polyhedron in order to distinguish this plane from the $z$-plane.

- Let $F \in C_{0}^{\infty}\left(\mathbb{C}^{n}\right)$ and its Taylor series is as in (1.4). We write $j_{N} F$ for the $N$-th order Taylor polynomial of $F$ at the origin, i.e.,

$$
j_{N} F(z, \bar{z})=\sum_{|\alpha+\beta| \leq N} C_{\alpha \beta} z^{\alpha} \bar{z}^{\beta}
$$


When $\left\{\xi \in \mathbb{R}_{>}^{n}:|\xi| \leq N\right\} \cap \mathcal{N}_{+}(F)=\emptyset$, set $j_{N} F \equiv 0$. The principal part $F_{0}$ of $F$ is the polynomial defined by

$$
F_{0}(z, \bar{z})=\sum_{\alpha+\beta \in \mathcal{N}(F)} C_{\alpha \beta} z^{\alpha} \bar{z}^{\beta}
$$

- We use the words pure terms for any harmonic polynomial and mixed terms for any sum of monomials that are neither holomorphic nor anti-holomorphic.

\section{Elementary properties of NeWton polyhedra}

2.1. Polyhedra. In order to treat delicate properties of Newton polyhedra, it is necessary to use many kinds of terminology concerning convex geometry. Refer to [42] for general theory of convex polyhedra.

For $(a, l) \in \mathbb{Z}^{n} \times \mathbb{Z}$, let $H(a, l)$ and $H_{+}(a, l)$ be a hyperplane and a closed halfspace in $\mathbb{R}^{n}$ defined by

$$
\begin{aligned}
& H(a, l):=\left\{\xi \in \mathbb{R}^{n}:\langle a, \xi\rangle=l\right\}, \\
& H_{+}(a, l):=\left\{\xi \in \mathbb{R}^{n}:\langle a, \xi\rangle \geq l\right\},
\end{aligned}
$$

respectively. A (convex) polyhedron is an intersection of closed halfspaces: a set $P \subset \mathbb{R}^{n}$ presented in the form

$$
P=\bigcap_{j=1}^{N} H_{+}\left(a^{j}, l_{j}\right)
$$

for some $a^{1}, \ldots, a^{N} \in \mathbb{Z}^{n}$ and $l_{1}, \ldots, l_{N} \in \mathbb{Z}$. A polyhedron $P$ is bounded if $P$ does not contain a ray $\{x+\lambda y: \lambda>0\}$ for any $x \in P$ and $y \neq 0$.

Let $P$ be a polyhedron in $\mathbb{R}^{n}$. A pair $(a, l) \in \mathbb{Z}^{n} \times \mathbb{Z}$ is said to be valid for $P$ if $P$ is contained in $H_{+}(a, l)$. A face of $P$ is any set of the form

$$
\kappa=P \cap H(a, l),
$$

where $(a, l)$ is valid for $P$. Since $(0,0)$ is always valid, we consider $P$ itself as a trivial face of $P$; the other faces are called proper faces. Considering the valid pair $(0,-1)$, we see that the empty set is always a face of $P$. Indeed, $H_{+}(0,-1)=\mathbb{R}^{n}$, but $H(0,-1)=\emptyset$. For a nonempty proper face $\kappa$ and a nonzero vector $a \in \mathbb{Z}^{n}$, we say that a determines $\kappa$ if $(a, l)$ is valid for $P$ and the equation (2.2) holds for some $l \in \mathbb{Z}$. Without any mentioning, the above vector $a=\left(a_{1}, \ldots, a_{n}\right)$ is always chosen to be primitive, i.e., $\operatorname{gcd}\left(a_{1}, \ldots, a_{n}\right)= \pm 1$. On the other hand, it is easy to see that any face is a polyhedron. The dimension of a face $\kappa$ is the dimension of its affine hull (i.e., the intersection of all affine flats that contain $\kappa)$. The faces of dimensions 0 and $\operatorname{dim}(P)-1$ are called vertices and facets, respectively. The vertices are denoted by boldfaces $\left(\mathbf{v}_{j}, \mathbf{e}_{j}\right.$, etc.).

For a subset $A$ of $\mathbb{R}^{n}$, the convex hull of $A$ is the intersection of all convex sets that contain $A$ :

$$
\operatorname{conv}(A):=\bigcap\left\{K \subset \mathbb{R}^{n}: A \subset K, K \text { convex }\right\}
$$


A proper face $\kappa$ of $P$ can be expressed as $\kappa=\operatorname{conv}\left(\left\{\mathbf{v}_{1}, \ldots, \mathbf{v}_{m}\right\}\right)$ where $\mathbf{v}_{1}, \ldots, \mathbf{v}_{m}$ are the vertices of $\kappa$.

2.2. Newton polyhedra. Let $F \in C_{0}^{\infty}\left(\mathbb{C}^{n}\right)$. The definition of the Newton polyhedron of $F$ has been given in the Introduction. Let us explain many useful properties of Newton polyhedra. The case when $F(0,0) \neq 0$ is trivial (when $\mathcal{N}_{+}(F)=\mathbb{R}_{>}^{n}$ and $\mathcal{N}(F)=\{0\})$. Hereafter in this paper, we always assume that $F(0,0)=0$.

Let $a \in \mathbb{N}_{0}^{n}$ and let $\kappa$ be a proper face of $\mathcal{N}_{+}(F)$. When " $a$ determines $\kappa$ ", we give some remarks about the relationship between $a$ and $\kappa$.

(i) For a given nonzero vector $a \in \mathbb{N}_{0}^{n}$, a proper face $\kappa$ of $\mathcal{N}_{+}(F)$ can be uniquely determined through the equation (2.2).

(ii) Conversely, it is easy to see that for a given proper face $\kappa$ of $\mathcal{N}_{+}(F)$, there may be many vectors $a \in \mathbb{N}_{0}^{n}$ determining $\kappa$ through (2.2). Note that $\kappa$ is a facet of $\mathcal{N}_{+}(F)$ if and only if the vector $a$ is uniquely determined by $\kappa$.

(iii) It will be shown in Lemma 2.1 that $\kappa$ is a bounded face if and only if $a$ belongs to $\mathbb{N}^{n}$ (i.e., each component of $a$ is positive).

It is known in [42] that Newton polyhedra are polyhedra, i.e., Newton polyhedra can be expressed as an intersection of finitely many closed half spaces. To be more precise, $\mathcal{N}_{+}(F)$ has the following properties.

Lemma 2.1. There exist finitely many valid pairs $\left(a_{j}, l_{j}\right) \in \mathbb{N}_{0}^{n} \times \mathbb{N}$ for $j=1, \ldots, m$ such that

$$
\mathcal{N}_{+}(F)=\bigcap_{j=1}^{m} H_{+}\left(a_{j}, l_{j}\right) .
$$

Furthermore, for a proper face $\kappa$ of the polyhedron $\mathcal{N}_{+}(F)$ defined by a valid pair $(a, l)$, the following three conditions are equivalent:

(i) The face $\kappa$ is bounded;

(ii) There are finitely many points in $\kappa \cap \mathbb{N}_{0}^{n}$;

(iii) Every component of a is positive (i.e., $a \in \mathbb{N}^{n}$ ).

Proof. It is easy to see that $\left(a_{j}, l_{j}\right)$ belong to $\mathbb{N}_{0}^{n} \times \mathbb{N}$ for $j=1, \ldots, m$.

Let us show the equivalence of the three conditions. Since the equivalence of (i) and (ii) is obvious, we will show the implications (iii) $\Rightarrow$ (i) and (ii) $\Rightarrow$ (iii).

$\left((\right.$ iii $) \Longrightarrow(\mathrm{i})$.) $\quad$ Let $\kappa$ be decided by the valid pair $(a, l) \in \mathbb{N}^{n} \times \mathbb{N}$. Since $a_{j}>0$ for all $j$, the set $H(a, l) \cap \mathbb{R}_{>}^{n}$ is bounded polyhedron containing $\kappa$, which implies the boundedness of $\kappa$.

((ii) $\Longrightarrow$ (iii).) Let us assume that the condition (iii) does not hold. Without loss of generality, we may set $a_{1}=0$. Since $\kappa=P \cap\left\{\xi: \sum_{j=2}^{n} a_{j} \xi_{j}=l\right\}$, if $\xi \in \mathbb{N}_{0}^{n}$ is contained in $\kappa$, then $\xi+\{(j, 0, \ldots, 0)\}$ is also contained in $\kappa$ for all $j \in \mathbb{N}$, which is a contradiction to (ii). 
For a bounded face $\kappa$ of $\mathcal{N}_{+}(F)$, the $\kappa$-part $F_{\kappa}$ of $F$ has a good homogeneity, which is useful for later investigation. For $z=\left(z_{1}, \ldots, z_{n}\right) \in \mathbb{C}^{n}, \zeta \in \mathbb{C}, a=\left(a_{1} \ldots, a_{n}\right) \in \mathbb{N}_{0}^{n}$, denote

$$
\zeta^{a} \bullet z:=\left(\zeta^{a_{1}} z_{1}, \ldots, \zeta^{a_{n}} z_{n}\right) .
$$

Lemma 2.2. Let $\kappa$ be a bounded face of $\mathcal{N}_{+}(F)$ defined by a valid pair $(a, l) \in \mathbb{N}^{n} \times \mathbb{N}$. Then, $F_{\kappa}$ has the quasihomogeneous property:

$$
F_{\kappa}\left(r^{a} \bullet z, \overline{r^{a} \bullet z}\right)=r^{l} F_{\kappa}(z, \bar{z}), \quad \text { for all } r \geq 0 .
$$

Furthermore, if $F_{\kappa}$ is holomorphic, then it has the following stronger property:

$$
F_{\kappa}\left(\zeta^{a} \bullet z\right)=\zeta^{l} F_{\kappa}(z), \quad \text { for all } \zeta \in \mathbb{C} .
$$

Proof. From the definition of the $\kappa$-part (1.6), we have

$$
\begin{gathered}
F_{\kappa}\left(r^{a} \bullet z, \overline{r^{a} \bullet z}\right)=\sum_{\alpha+\beta \in \kappa} C_{\alpha \beta} \cdot \prod_{j=1}^{n}\left(r^{a_{j}} z_{j}\right)^{\alpha_{j}} \cdot \prod_{j=1}^{n}\left(r^{a_{j}} \bar{z}_{j}\right)^{\beta_{j}} \\
=r^{\langle a, \alpha+\beta\rangle}\left(\sum_{\alpha+\beta \in \kappa} C_{\alpha \beta} z^{\alpha} \bar{z}^{\beta}\right)=r^{\langle a, \alpha+\beta\rangle} F_{\kappa}(z, \bar{z}) .
\end{gathered}
$$

Since the face $\kappa$ is determined by $H(a, l)$, the equality: $\langle a, \alpha+\beta\rangle=l$ always holds if $\alpha+\beta \in \kappa$. This implies (2.4).

When $f$ is holomorphic, $\beta_{j}=0$ in (2.6), so the equation (2.5) can be samely shown.

Newton polyhedra keep some properties after being multiplied by non-zero functions.

Lemma 2.3. Let $h \in C_{0}^{\infty}\left(\mathbb{C}^{n}\right)$ satisfy $h(0,0) \neq 0$. Then we have

(i) $\mathcal{N}_{+}(F)=\mathcal{N}_{+}(h F)$;

(ii) For a bounded face $\kappa$ of $\mathcal{N}_{+}(F)$, the $\kappa$-part of the function $h(z, \bar{z}) F(z, \bar{z})$ is $h(0,0) F_{\kappa}(z, \bar{z})$.

Proof. For general $g \in C_{0}^{\infty}\left(\mathbb{C}^{n}\right)$, it is easy to see that $\mathcal{N}_{+}(g F) \subset \mathcal{N}_{+}(F)$. By regarding vertices as bounded faces, this inclusion shows that (ii) implies (i). Therefore, we only show (ii).

Let $(a, l)$ be a valid pair defining $\kappa$. Then the boundedness of $\kappa$ implies that every component of $a$ is positive (see Lemma 2.1). The positivity of $a$ and Taylor's formula imply that

$$
\begin{aligned}
& h(z, \bar{z})=h(0,0)+\sum_{j=1}^{n} z_{j} h_{j}(z, \bar{z})+\sum_{j=1}^{n} \bar{z}_{j} \overline{h_{j}(z, \bar{z})} \\
& F(z, \bar{z})=F_{\kappa}(z, \bar{z})+R_{\kappa}(z, \bar{z})
\end{aligned}
$$


where $h_{j}, \bar{h}_{j} \in C_{0}^{\infty}\left(\mathbb{C}^{n}\right)$ and $R_{\kappa} \in C_{0}^{\infty}\left(\mathbb{C}^{n}\right)$ satisfying that $\mathcal{N}_{+}\left(R_{\kappa}\right) \subset H_{+}(a, l+1)$ with a valid pair $(a, l)$ defining $\kappa$. From (2.7), noticing that $\mathcal{N}_{+}\left(z_{j} h_{j} F_{\kappa}\right), \mathcal{N}_{+}\left(\bar{z}_{j} \bar{h}_{j} F_{\kappa}\right) \subset$ $H^{+}(a, l+1)$ from the positivity of $a$, we have

$$
\mathcal{N}_{+}\left(h F-h(0) F_{\kappa}\right) \subset H^{+}(a, l+1),
$$

which shows (ii).

2.3. Some quantities related to Newton polyhedra. Let $F \in C_{0}^{\infty}\left(\mathbb{C}^{n}\right)$. When $\mathcal{N}_{+}(F)$ meets the $\xi_{j}$-axis, let $\rho_{j}(F)$ be the coordinate of the point at which the Newton diagram $\mathcal{N}(F)$ intersect the $\xi_{j}$-axis, that is,

$$
\rho_{j}(F)=\min \left\{\xi_{j}>0:\left(0, \ldots, 0, \xi_{j}, 0, \ldots, 0\right) \in \mathcal{N}_{+}(F)\right\} .
$$

If $\mathcal{N}_{+}(F)$ does not meet the $\xi_{j}$-axis, then we set $\rho_{j}(F):=\infty$. The $n$-tuple of numbers $\rho(F)$ is defined by

$$
\rho(F)=\left(\rho_{1}(F), \ldots, \rho_{n}(F)\right) \in(\mathbb{N} \cup\{\infty\})^{n} .
$$

(When $F$ is convenient, the definition of $\rho_{j}$ is the same as it in the Introduction.)

In the special cases of $F$, the following are easy to see.

- If $F$ is flat, then $\rho(F)=(\infty, \ldots, \infty)$.

- $F$ is convenient if and only if every $\rho_{j}(F)$ is a positive integer $(<\infty)$.

2.4. Newton polyhedra associated to real hypersurfaces. Let $M$ be a smooth real hypersurface in $\mathbb{C}^{n}$ and let $p$ lie on $M$. Let $(z)$ be a holomorphic coordinate around $p$ such that $p=0$ and let $r$ be a local defining function for $M$ near $p$ on the coordinate $(z)$. We respectively define the Newton polyhedron and the Newton diagram with respect to $(M, p ;(z))$ by

$$
\mathcal{N}_{+}(M, p ;(z)):=\mathcal{N}_{+}(r), \quad \mathcal{N}(M, p ;(z)):=\mathcal{N}(r) .
$$

Moreover, the $n$-tuple of numbers $\rho(M, p ;(z))$ is defined by

$$
\rho(M, p ;(z))=\left(\rho_{1}(M, p ;(z)), \ldots, \rho_{n}(M, p ;(z))\right) \in(\mathbb{N} \cup\{\infty\})^{n}
$$

with $\rho_{j}(M, p ;(z))=\rho_{j}(r)$ for $j=1, \ldots, n$. We remark that these are well-defined. Indeed, since another defining function for $M$ at $p$ can be written as $h(z, \bar{z}) r(z, \bar{z})$ with a positive $C^{\infty}$ function $h$, Lemma 2.3 (i) implies that the shape of the Newton polyhedron is independent of the choice of defining functions after fixing the coordinates. In particular, $\rho_{1}(M, p ;(z))$ has the same property.

By changing the order of variables if necessary, we can always choose a coordinate (z) such that

$$
\rho_{1}(M, p ;(z)) \geq \cdots \geq \rho_{n}(M, p ;(z)) .
$$

Hereafter in this paper, we only consider these coordinates without any mentioning. It is easy to see the following:

(i) $\rho_{n}(M, p ;(z))=1$. 
(ii) A defining function for $M$ is convenient on the coordinate $(z)$ if and only if $\rho_{1}(M, p ;(z))<\infty$.

\section{REMARKS ON NONDEGENERACY CONDITION}

Let $F \in C_{0}^{\infty}\left(\mathbb{C}^{n}\right)$. The definition of the nondegeneracy condition on $F$ has been given in the Introduction. Since this condition plays important roles in our analysis, its useful properties will be precisely explained.

The following four sets of compex curves are defined, which will be often used in this paper.

$$
\begin{aligned}
& \Gamma:=\left\{\gamma=\left(\gamma_{1}, \ldots, \gamma_{n}\right): \gamma_{j} \in \mathcal{O}_{0}(\mathbb{C}), \gamma_{j}(0)=0 \text { for } j=1, \ldots, n\right\} \backslash\{0\}, \\
& \Gamma^{*}:=\left\{\gamma=\left(\gamma_{1}, \ldots, \gamma_{n}\right) \in \Gamma: \gamma_{j} \not \equiv 0 \text { for } j=1, \ldots, n\right\}, \\
& \widetilde{\Gamma}^{*}:=\left\{\left(c_{1} t^{a_{1}}, \ldots, c_{n} t^{a_{n}}\right) \in \Gamma^{*}: c \in\left(\mathbb{C}^{*}\right)^{n}, a \in \mathbb{N}^{n}, t \in \mathbb{C}\right\}, \\
& \widetilde{\Gamma}_{\kappa}^{*}:=\left\{\left(c_{1} t^{a_{1}}, \ldots, c_{n} t^{a_{n}}\right) \in \widetilde{\Gamma}^{*}: a \text { determines } \kappa\right\},
\end{aligned}
$$

where $c=\left(c_{1}, \ldots, c_{n}\right) \in\left(\mathbb{C}^{*}\right)^{n}, a=\left(a_{1}, \ldots, a_{n}\right) \in \mathbb{N}^{n}$ and $\kappa$ is a bounded face of $\mathcal{N}_{+}(F)$ ( $\Gamma$ with $p=0$ and $\widetilde{\Gamma}_{\kappa}^{*}$ are the same as those in the Introduction).

3.1. Remarks on Definition 1.2. There are many remarks on the definition of nondegeneracy condition.

(1) By using the map $\Phi_{*}$ defined below as in (7.5), the set $\widetilde{\Gamma}_{\kappa}^{*}$ is simply expressed as $\widetilde{\Gamma}_{\kappa}^{*}=\left\{\gamma \in \widetilde{\Gamma}^{*}: \Phi_{*}(\gamma)=\kappa\right\}$.

(2) The set $\widetilde{\Gamma}_{\kappa}^{*}$ seems complicated, but it often suffices to check the following conditions to see the nondegeneracy of $F_{\kappa}$ :

(a) The restriction of the zero variety:

$$
V\left(F_{\kappa}\right):=\left\{z \in \mathbb{C}^{n}: F_{\kappa}(z, \bar{z})=0\right\}
$$

to $\left(\mathbb{C}^{*}\right)^{n} \cup\{0\}$ does not contain any complex curves through the origin;

(b) $F_{\kappa} \circ \gamma \not \equiv 0$ for any $\gamma \in \Gamma^{*}$;

(c) $F_{\kappa} \circ \gamma \not \equiv 0$ for any $\gamma \in \widetilde{\Gamma}^{*}$.

By Local Parametrization theorem ([17]), (a) and (b) are equivalent. Moreover, the following implications hold: $(\mathrm{b}) \Longrightarrow(\mathrm{c}) \Longrightarrow$ (5.1).

(3) In order to check the nondegeneracy condition for a given $F$, one must consider not only the facets (i.e., $(n-1)$-dimensional faces) but also arbitrary dimensional faces of the Newton polyhedron $\mathcal{N}_{+}(F)$. For example, let us consider the case of the function: $F(z, \bar{z})=\left|z_{1}\right|^{4}-2\left|z_{1} z_{2}\right|^{2}+\left|z_{2}\right|^{4}+\left|z_{3}\right|^{4} \in C_{0}^{\infty}\left(\mathbb{C}^{3}\right)$. Let

$$
\kappa_{1}:=\operatorname{conv}\left(\left\{4 \mathbf{e}_{1}, 4 \mathbf{e}_{2}, 4 \mathbf{e}_{3}\right\}\right) \text { and } \kappa_{2}:=\operatorname{conv}\left(\left\{4 \mathbf{e}_{1}, 4 \mathbf{e}_{2}\right\}\right) .
$$

It is easy to see that $\kappa_{1}$ is the facet of $\mathcal{N}_{+}(F)$ and $F_{\kappa_{1}}$ is nondegenerate; while $\kappa_{2}$ is a one-dimensional face of $\mathcal{N}_{+}(F)$ and $F_{\kappa_{1}}$ is degenerate. Thus, $F$ is degenerate. 
(4) In the definition of the nondegeneracy condition, $F_{\kappa}$ may vanish at some set in $\left(\mathbb{C}^{*}\right)^{n}$ which does not have the complex structure. For example, let us consider the $C^{\infty}$ functions:

$$
\begin{aligned}
& F_{1}(z, \bar{z})=|z|^{8}+\frac{15}{7}|z|^{2} \operatorname{Re}\left(z^{6}\right) \quad \text { on } \mathbb{C} \\
& F_{2}(z, \bar{z})=\left|z_{1}\right|^{8}+\frac{15}{7}\left|z_{1}\right|^{2} \operatorname{Re}\left(z_{1}^{6}\right)+\left|z_{2}\right|^{8}+\frac{15}{7}\left|z_{2}\right|^{2} \operatorname{Re}\left(z_{2}^{6}\right) \quad \text { on } \mathbb{C}^{2} .
\end{aligned}
$$

They vanish on some sets in $\mathbb{C}^{*}$ or $\left(\mathbb{C}^{*}\right)^{2}$, but these sets contain no complex curves. The function $F_{1}$ appears in a famous example of Kohn-Nirenberg in [25]. Note that the above functions are real-valued plurisubharmonic functions.

(5) Let us recall the definition of the nondegeneracy condition introduced by Kouchnirenko [27], which plays important roles in the study of singularity theory (see [1], [33]). Let $F$ be a holomorphic function defined near the origin in $\mathbb{C}^{n}$. The function $F$ is said to be nondegenerate in the sense of Kouchnirenko if

$$
\left(\frac{\partial F_{\kappa}}{\partial z_{1}}, \cdots, \frac{\partial F_{\kappa}}{\partial z_{n}}\right) \neq 0 \text { on }\left(\mathbb{C}^{*}\right)^{n}
$$

for any bounded face $\kappa$ of $\mathcal{N}_{+}(F)$. The following Euler identity equation is followed from the equation (2.5) : $\sum_{j=1}^{n} a_{j} z_{j} \frac{\partial F_{\kappa}}{\partial z_{j}}=l F_{\kappa}$ for $z \in \mathbb{C}^{n}$, where $(a, l) \in \mathbb{N}^{n} \times \mathbb{N}$ is a valid pair determining the face $\kappa$. From this equation, if $\frac{\partial F_{\kappa}}{\partial z_{j}}$ has a common zero on $\left(\mathbb{C}^{*}\right)^{n}$ for $j=1, \ldots, n$, then $F_{\kappa}$ vanishes there. Therefore, our nondegeneracy condition implies that of Kouchnirenko. But, it follows from Lemma 3.4, below, that almost all holomorphic functions are degenerate in our sense, so this implication is not useful. In other words, our nondegeneracy condition makes sense only in the mixed variables case.

(6) The notion "nondegeneracy condition" in Definition 1.2 is not invariant under biholomorphic maps. For example, $\left|z_{1}\right|^{2}+\left|z_{2}\right|^{4}$ is nondegenerate, but $\left|z_{1}-z_{2}\right|^{2}+\left|z_{2}\right|^{4}$ is degenerate. Note that Kouchnirenko's nondegeneracy is also in the same situation. Consider the functions: $z_{1}^{2}+z_{2}^{4}$ and $\left(z_{1}-z_{2}\right)^{2}+z_{2}^{4}$. Therefore, when the chosen coordinate should be strongly paid attention, we write " $F$ is nondegenerate on the coordinate $(z) . "$

3.2. Elementary properties of nondegeneracy conditions. The nondegeneracy property remains after being multiplied by nonzero functions.

Lemma 3.1. Let $h$ be a real-valued smooth function with $h(0,0) \neq 0$. If $F$ is nondegenerate, then so is $h(z, \bar{z}) F(z, \bar{z})$.

Proof. This is trivial from Lemma 2.3 (ii).

Remark 3.2. If there exists a canonical coordinate $(z)$ for $M$ at $p$, then every local defining function for $M$ is nondegenerate on $(z)$. 
The one-dimensional case is obvious.

Lemma 3.3. Every nonflat smooth function $F$ of one variable is nondegenerate.

Proof. Let us assume that $F$ is degenerate. Then the principal part $F_{0}$ of $F$ (see (1.15) $)$ must identically equal zero, which is a contradiction.

More generally, let us consider the case of the $\mathbf{v}$-part of $F$, where $\mathbf{v}$ is a vertex of $\mathcal{N}_{+}(F)$. In the one-dimensional case, $F_{\mathbf{v}}$ is always nondegenerate from the above lemma. But, $F_{\mathbf{v}}$ may be degenerate in the multi-dimensional case. Indeed, consider the two-dimensional example: $F_{\mathbf{v}}(z, \bar{z})=z_{1}^{2} \bar{z}_{1}^{8} z_{2}^{6} \bar{z}_{2}^{4}-z_{1}^{4} \bar{z}_{1}^{6} z_{2}^{4} \bar{z}_{2}^{6}$. Note that $\mathbf{v}=\{(10,10)\}$ and $F_{\mathbf{v}} \circ \gamma \equiv 0$, where $\gamma(t)=(t, t)$.

The following lemma shows that there are few pluriharmonic functions satisfying the nondegeneracy condition.

Lemma 3.4. Let $F$ be pluriharmonic near the origin. Then the following three conditions are equivalent.

(i) $F$ is nondegenerate;

(ii) The Newton diagram $\mathcal{N}(F)$ consists of only one vertex in $\mathbb{N}_{0}^{n}$;

(iii) There exist $C_{1}, \ldots, C_{n} \in \mathbb{N}_{0}$ such that $\mathcal{N}_{+}(F)=\left\{\xi \in \mathbb{R}_{\geq}^{n}: \xi_{j} \geq C_{j}\right.$ for $\left.j=1, \ldots, n\right\}$.

In particular, when $F$ is holomorphic or antiholomorphic, the same equivalences are established.

Proof. It suffices to consider the case when $F$ is a holomorphic function. Since the implications (ii) $\Longrightarrow$ (i) and (ii) $\Longleftrightarrow$ (iii) are obvious, we only show that the implication (i) $\Longrightarrow$ (ii).

Let us assume that (ii) does not hold. Then, $\mathcal{N}_{+}(F)$ has a one-dimensional bounded face $\kappa$. It is easy to see that $F_{\kappa}$ vanishes at some point $c=\left(c_{1} \ldots, c_{n}\right) \in\left(\mathbb{C}^{*}\right)^{n}$. It follows from the equation (2.5) in Lemma 2.2 that $F_{\kappa}\left(c_{1} t^{a_{1}}, \ldots, c_{n} t^{a_{n}}\right)=0$ for any $t \in \mathbb{C}$ where $a=\left(a_{1}, \ldots, a_{n}\right) \in \mathbb{N}^{n}$ determines $\kappa$, which implies that $F$ is not nondegenerate.

From the definition of the nondegeneracy, it is important to understand the geometrical properties of the singular varieties $V\left(F_{\kappa}\right)$ in (3.2). The following is an interesting result about $V\left(F_{\kappa}\right)$.

Lemma $3.5([2])$. Let $\kappa$ be a bounded face of $\Gamma_{+}(F)$. If $F_{\kappa}$ is plurisubharmonic on $\mathbb{C}^{2}$, then $V\left(F_{\kappa}\right)$ contains only finitely many complex curves.

\section{A NEW INVARIANT AND ADAPTED COORDINATES}

In this section, we introduce a new invariant and coordinates related to this invariant, which will be intrinsically important in this paper. 
4.1. The invariant $\rho_{1}(M, p)$. From the definition of $\rho_{1}(M, p ;(z))$, we can naturally define the following invariant:

$$
\rho_{1}(M, p)=\sup _{(z)}\left\{\rho_{1}(M, p ;(z))\right\},
$$

where the supremum is taken over all holomorphic coordinates $(z)$ around $p$. A given holomorphic coordinate $(z)$ at $p$ is called an adapted coordinate for $M$ at $p$ if the following equality holds:

$$
\rho_{1}(M, p ;(z))=\rho_{1}(M, p) .
$$

When $\rho_{1}(M, p)$ is finite, there exists an adapted coordinate for $M$ at $p$ since $\rho_{1}(M, p ;(z))$ takes a positive integer. But, when $\rho_{1}(M, p)=\infty$, there does not always exist an adapted coordinate. Indeed, let us consider the case of the real hypersurface $M \subset \mathbb{C}^{2}$ in [3], [13] defined by $\operatorname{Re}\left(z_{2}\right)+F\left(z_{1}, \bar{z}_{1}\right)=0$ where $F \in C_{0}^{\infty}(\mathbb{C})$ admits the Taylor series $\sum_{j=2}^{\infty} j ! \operatorname{Re}\left(z_{1}^{j}\right)$. It can be easily seen that $\rho_{1}(M, 0)=\infty$ but $\rho_{1}(M, 0 ;(z))<\infty$ for any holomorphic coordinates $(z)$ at the origin. Note that regardless of the value of $\rho_{1}(M, p)$, canonical coordinates in Theorem 1.3 are always adapted coordinates.

Let us consider the relationship between the invariant (4.1) and the regular type of $M$ at $p$ (see (1.2)). From the definition, it is easy to see the inequality: $\rho_{1}(M, p ;(z)) \leq$ $\Delta_{1}^{\text {reg }}(M, p)$ for any coordinates $(z)$. Thus, the following inequality always holds:

$$
\rho_{1}(M, p) \leq \Delta_{1}^{\mathrm{reg}}(M, p) .
$$

Furthermore, we show that these two invariants always agree.

Proposition 4.1. $\rho_{1}(M, p)=\Delta_{1}^{\mathrm{reg}}(M, p)$.

Proof. From (4.3), it suffices to show that $\rho_{1}(M, p) \geq \Delta_{1}^{\text {reg }}(M, p)$. We may assume that $p$ is the origin.

First, let us consider the case when $\Delta_{1}^{\mathrm{reg}}(M, p)<\infty$. Since ord $(r \circ \gamma)$ is an integer, there exists a regular holomorphic mapping $\gamma \in \Gamma_{\text {reg }}$ attaining $\Delta_{1}^{\text {reg }}(M, p)=\operatorname{ord}(r \circ$ $\gamma)$. By the implicit function theorem, without loss of generality, the map $\gamma$ may be expressed by $z_{1}=t, z_{j}=\varphi_{j}(t)(j=2, \ldots, n)$, where $\varphi_{j} \in \mathcal{O}_{0}(\mathbb{C})$ with $\varphi_{j}(0)=0$. Let $(w)=\left(w_{1}, \ldots, w_{n}\right)$ be the holomorphic coordinate defined by $w_{1}=z_{1}, w_{j}=$ $z_{j}-\varphi_{j}\left(z_{1}\right)(j=2, \ldots, n)$. We denote $\varphi\left(w_{1}\right):=\left(\varphi_{1}\left(w_{1}\right), \ldots, \varphi_{n}\left(w_{1}\right)\right)$. Let $\tilde{r}$ be defined by $\tilde{r}(w, \bar{w})=r\left(w+\varphi\left(w_{1}\right), \overline{w+\varphi\left(w_{1}\right)}\right)$, which is a local defining function for $M$ near $p$ on the coordinate $(w)$. Let $\gamma_{*}(t):=(t, 0, \ldots, 0) \in \Gamma$. Then we can see $\tilde{r}\left(\gamma_{*}(t), \overline{\gamma_{*}(t)}\right)=$ $r(\gamma(t), \overline{\gamma(t)})$. Since $\operatorname{ord}\left(\tilde{r} \circ \gamma_{*}\right)=\operatorname{ord}(r \circ \gamma)$, we have

$$
\rho_{1}(M, p) \geq \rho_{1}(M, p ;(w))=\operatorname{ord}\left(\tilde{r} \circ \gamma_{*}\right)=\operatorname{ord}(r \circ \gamma)=\Delta_{1}^{\mathrm{reg}}(M, p) .
$$

Next, let us consider the case when $\Delta_{1}^{\text {reg }}(M, p)=\infty$. For any $N \in \mathbb{N}$, there exists a $\gamma_{N} \in \Gamma_{\text {reg }}$ such that ord $\left(r \circ \gamma_{N}\right) \geq N$. In a similar fashion to the above case of finite $\Delta_{1}^{\text {reg }}(M, p)$, we can show that, for any $N \in \mathbb{N}$, there exists a holomorphic coordinate 
$(w)$ such that $\rho_{1}(M, p ;(w)) \geq N$. This means that $\rho_{1}(M, p) \geq N$ for any $N \in \mathbb{N}$, which implies that $\rho_{1}(M, p)=\infty$.

4.2. Properties of adapted coordinates. Let us consider a necessary condition for the adaptedness of coordinates. This condition will be useful for the investigation of the regular and singular types in Section 14.

Hereafter in this section, we assume that $F \in C_{0}^{\infty}\left(\mathbb{C}^{n}\right)$ is convenient (i.e. $\rho_{j}(F)<\infty$ for $j=1, \ldots, n)$. Without loss of generality, we may assume that $\rho_{1}(F)=\max \left\{\rho_{j}(F)\right.$ : $j=1, \ldots, n\}$. Let $\mathbf{v}_{1}^{*}:=\left(\rho_{1}(F), 0, \ldots, 0\right)$, which is the vertex of $\mathcal{N}_{+}(F)$ intersecting the $\xi_{1}$-axis.

Lemma 4.2. Suppose that there exist a bounded face $\kappa$ of $\mathcal{N}_{+}(F)$ and the complex curve $\gamma \in \widetilde{\Gamma}_{\kappa}^{*}$ (see (3.1)) with $\operatorname{ord}(\gamma)=1$ such that $\kappa$ contains the vertex $\mathbf{v}_{1}^{*}$ and $F_{\kappa} \circ \gamma \equiv 0$. Then there exists a local biholomorphic mapping $\Psi:\left(\mathbb{C}^{n}, 0\right) \rightarrow\left(\mathbb{C}^{n}, 0\right)$ such that $\rho_{1}(F \circ \Psi)>\rho_{1}(F)$.

Proof. Without loss of generality, we assume that there exists a holomorphic mapping: $\gamma(t)=\left(t, c_{2} t^{a_{2}}, \ldots, c_{n} t^{a_{n}}\right)$ with $c_{j} \in \mathbb{C}^{*}$ and $a_{j} \in \mathbb{N}$ for $j=2, \ldots, n$ such that $\gamma \in \widetilde{\Gamma}_{\kappa}^{*}$ and $F_{\kappa} \circ \gamma \equiv 0$ on $\mathbb{C}$. Lemma 7.1, below, implies

$$
(F \circ \gamma)(t, \bar{t})=\left(F_{\kappa} \circ \gamma\right)(t, \bar{t})+R_{\kappa}(t, \bar{t})=R_{\kappa}(t, \bar{t}),
$$

where $R_{\kappa} \in \widetilde{\mathcal{H}}_{l+1}$ with $l=\rho_{1}(F)$. (Note that $\Phi(\gamma)=\Phi_{*}(\gamma)=\kappa$.)

Now, let us define the local biholomorphic mapping: $z=\Psi(w)$ as $z_{1}=w_{1}, z_{j}=$ $w_{j}+c_{j} w_{1}^{a_{j}}(j=2, \ldots, n)$. Let $\gamma_{*}(t):=(t, 0, \ldots, 0) \in \Gamma$. Since $\Psi \circ \gamma_{*}=\gamma$, (4.4) gives

$$
(F \circ \Psi)\left(\gamma_{*}(t), \overline{\gamma_{*}(t)}\right)=(F \circ \gamma)(t, \bar{t})=R_{\kappa}(t, \bar{t}) .
$$

Therefore, we can see the following.

$$
\rho_{1}(F \circ \Psi)=\operatorname{ord}\left(F \circ \Psi \circ \gamma_{*}\right)=\operatorname{ord}\left(R_{\kappa}\right) \geq l+1>\rho_{1}(F) .
$$

Definition 4.3. Let $\kappa$ be a bounded face of a polyhedron $P \subset \mathbb{R}_{>}^{n}$. We call $\kappa$ a regular face of $P$ if $\kappa$ is determined by a vector $a=\left(a_{1}, \ldots, a_{n}\right) \in \mathbb{N}^{n}$ satisfying that $\min \left\{a_{j}: j=1, \ldots, n\right\}=1$.

Let $r$ be a convenient local defining function for $M$ at $p$ on the coordinate $(z)$. Let $m$ be the maximum integer such that $\rho_{j}(r)=\rho_{1}(r)$ for $j=1, \ldots, m$. Let $\mathcal{V}$ be the set of vertices of $\mathcal{N}_{+}(r)$ defined by $\mathcal{V}=\left\{\left(0, \ldots, \rho_{1}(r), \ldots, 0\right): j=1, \ldots, m\right\}$.

Lemma 4.4. If $(z)$ is an adapted coordinate for $M$ at $p$, then $r_{\kappa}$ is nondegenerate for any regular face $\kappa$ of $\mathcal{N}_{+}(M, p ;(z))$ intersecting $\mathcal{V}$.

Proof. This can be easily shown by using Lemma 4.2 .

As a corollary of Lemma 4.4, we can see the following. 
Lemma 4.5. Let $(z)$ be an adapted coordinate for $M$ at $p$. Suppose that the Newton diagram $\mathcal{N}(M, p ;(z))$ consists of only one facet and that it is a regular face. Then $r_{\kappa}$ is nondegenerate for each face $\kappa$ of $\mathcal{N}_{+}(M, p ;(z))$ intersecting $\mathcal{V}$.

Proof. From Lemma 4.4, it suffices to check that all proper faces of $\mathcal{N}(M, p ;(z))$ are regular, which is easy.

\section{Proof of Theorem 1.1}

We are now in a position to prove Theorem 1.1.

From Proposition 4.1, it is easy to see that the inequalities:

$$
\Delta_{1}(M, p) \geq \Delta_{1}^{\mathrm{reg}}(M, p)=\rho_{1}(M, p) \geq \rho_{1}(M, p ;(z))
$$

always hold for any holomorphic coordinates $(z)$ at $p$. Recalling that an adapted coordinate $(z)$ at $p$ always exists if $\rho_{1}(M, p)<\infty$, we can see that the equality in (i) implies (ii) from (5.1). The implication: (ii) $\Longrightarrow$ (i) is obvious from (5.1).

Remark 5.1. In Theorem 1.1, the assumption: $\Delta_{1}^{\text {reg }}(M, p)<\infty$ can be weakened by the condition: an adapted coordinate exists for $M$ at $p$.

\section{ORDER OF VANISHING}

Let $F \in C_{0}^{\infty}\left(\mathbb{C}^{n}\right)$ with $F(0,0)=0$ and let $\Gamma$ be as in (3.1)

The order of vanishing of a nonflat function $F$ is defined by

$$
\begin{aligned}
& \operatorname{ord}(F):=\min \left\{l \in \mathbb{N}: \exists(\alpha, \beta) \in \mathbb{N}_{0}^{n} \times \mathbb{N}_{0}^{n}\right. \\
& \text { with } \left.|\alpha|+|\beta|=l \text { such that } D^{\alpha} \bar{D}^{\beta} F(0,0) \neq 0\right\} .
\end{aligned}
$$

When $F$ is flat, we set $\operatorname{ord}(F):=\infty$. For a vector-valued mapping $f=\left(f_{1}, \ldots, f_{m}\right) \in$ $\left(C_{0}^{\infty}(\mathbb{C})\right)^{m}$, let

$$
\operatorname{ord}(f):=\min \left\{\operatorname{ord}\left(f_{j}\right): j=1, \ldots, m\right\} .
$$

We remark that ord $(\gamma)$ is a positive integer for $\gamma \in \Gamma$. (Recall that $\Gamma$ does not contain the constant maps.)

For $l \in \mathbb{Z}_{\geq}$, let $\mathcal{H}_{l}$ be the subset of $\mathbb{C}[t, \bar{t}]$ defined by

$$
\mathcal{H}_{l}=\left\{\sum c_{j k} t^{j} \bar{t}^{k}: j, k \in \mathbb{N}_{0} \text { with } j+k=l \text { and } c_{j k} \in \mathbb{C}\right\}
$$

and let $\widetilde{\mathcal{H}}_{l}$ be the subset of $C_{0}^{\infty}(\mathbb{C})$ defined by

$$
\widetilde{\mathcal{H}}_{l}=\left\{\sum t^{j} \bar{t}^{k} a_{j k}(t, \bar{t}): j, k \in \mathbb{N}_{0} \text { with } j+k=l \text { and } a_{j k} \in C_{0}^{\infty}(\mathbb{C})\right\} .
$$

The following properties of the two classes can be directly seen. Let $l, l_{1}, l_{2} \in \mathbb{Z}_{\geq}$.

- $\mathcal{H}_{0}=\mathbb{C}$ and $\widetilde{\mathcal{H}}_{0}=C_{0}^{\infty}(\mathbb{C})$.

- $\mathcal{H}_{l} \subset \widetilde{\mathcal{H}}_{l}$. If $l_{1} \leq l_{2}$, then $\widetilde{\mathcal{H}}_{l_{2}} \subset \widetilde{\mathcal{H}}_{l_{1}}$.

- $\mathcal{H}_{l}$ and $\widetilde{\mathcal{H}}_{l}$ are vector spaces. Moreover, $\widetilde{\mathcal{H}}_{l}$ is an ideal of $C_{0}^{\infty}(\mathbb{C})$. 
- If $f_{1} \in \mathcal{H}_{l_{1}}$ and $f_{2} \in \mathcal{H}_{l_{2}}$, then $f_{1} f_{2} \in \mathcal{H}_{l_{1}+l_{2}}$.

- If $f_{1} \in \widetilde{\mathcal{H}}_{l_{1}}$ and $f_{2} \in \widetilde{\mathcal{H}}_{l_{2}}$ with $l_{1} \leq l_{2}$, then $f_{1}+f_{2} \in \widetilde{\mathcal{H}}_{l_{1}}$ and $f_{1} f_{2} \in \widetilde{\mathcal{H}}_{l_{1}+l_{2}}$.

- If $f \in \mathcal{H}_{l}$, then the following two conditions are equivalent:

$$
\text { (a) } \operatorname{ord}(f)=l, \quad(\mathrm{~b}) f \neq 0 \text {. }
$$

- If $f \in C_{0}^{\infty}(\mathbb{C})$ with $f(0,0)=0$, then the following three conditions are equivalent:
(a) $f \in \widetilde{\mathcal{H}}_{l}$,
(b) ord $(f) \geq l$,
(c) $f(t)=O\left(|t|^{l}\right)$.

\section{Asymptotics of $F \circ \gamma$}

Let $F \in C_{0}^{\infty}\left(\mathbb{C}^{n}\right)$ with $F(0,0)=0$ and let $\Gamma$ be as in $(3.1)$.

In this section, we compute the leading term of the asymptotic expansion of $F \circ \gamma$ for $\gamma \in \Gamma$ with respect to the classes $\mathcal{H}_{l}$ for $l \in \mathbb{N}_{0}$. This leading term can be determined by appropriate truncations of $F$ and $\gamma$.

The computation is divided into the two cases. One of them is the case when $\gamma$ belongs to $\Gamma^{*}$ (see (3.1)). This case is considered as a generic one. The second case treats the curves which are contained in some coordinate planes. (Note that the first case may be contained in the second one.) The second case seems very complicated but this complexity is not essential. Essential analytic ideas of the computation can be explained by using the first generic case only.

7.1. Asymptotics of $F \circ \gamma$ in the generic case. Let $\mathcal{F}_{F}^{C}$ denote the set of bounded faces of $\mathcal{N}_{+}(F)$. Exactly understanding the relationship between $\Gamma^{*}$ and $\mathcal{F}_{F}^{C}$ is the most important to compute the asymptotics of $F \circ \gamma$ for $\gamma \in \Gamma^{*}$.

Suppose that $F$ is not flat. We define the two important maps $l_{*}: \mathbb{N}^{n} \rightarrow \mathbb{N}$ and $\kappa_{*}: \mathbb{N}^{n} \rightarrow \mathcal{F}_{F}^{C}$ as follows:

$$
\begin{aligned}
& l_{*}(a):=\min \left\{\langle a, \xi\rangle: \xi \in \mathcal{N}_{+}(F)\right\}, \\
& \kappa_{*}(a):=\left\{\xi \in \mathcal{N}_{+}(F):\langle a, \xi\rangle=l_{*}(a)\right\}\left(=H\left(a, l_{*}(a)\right) \cap \mathcal{N}_{+}(F)\right) .
\end{aligned}
$$

We remark that $\kappa_{*}(a)$ is bounded for $a \in \mathbb{N}^{n}$ from Lemma 2.1.

Let us consider the sequence:

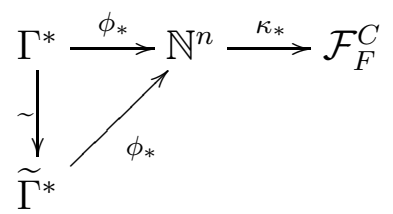

where $\Gamma^{*}, \widetilde{\Gamma}^{*}$ are as in (3.1) and the mappings $\tilde{\bullet}, \phi_{*}$ are defined as follows.

- Since $\gamma_{j} \not \equiv 0$ for $j=1, \ldots, n$ and $\gamma_{j}(0)=0, \gamma_{j}(t)$ can be expressed by the convergence series expansion of $t$ as $\gamma_{j}(t)=c_{j} t^{a_{j}}+\cdots$ with some $a_{j} \in \mathbb{N}$ and 
$c_{j} \in \mathbb{C}^{*}$ for $j=1, \ldots, n$. Then let $\tilde{\gamma}_{j}$ be the monomial defined by $\tilde{\gamma}_{j}(t)=c_{j} t^{a_{j}}$. The map $\widetilde{r}$ from $\Gamma^{*}$ to $\widetilde{\Gamma}^{*}$ is defined as follows: for $\gamma \in \Gamma^{*}$,

$$
\tilde{\gamma}(t)=\left(\tilde{\gamma}_{1}(t), \ldots, \tilde{\gamma}_{n}(t)\right)=\left(c_{1} t^{a_{1}}, \ldots, c_{n} t^{a_{n}}\right) \in \widetilde{\Gamma}^{*} .
$$

- The map $\phi_{*}: \Gamma^{*} \rightarrow \mathbb{N}^{n}$ is defined by

$$
\phi_{*}(\gamma)=\left(\operatorname{ord}\left(\gamma_{1}\right), \ldots, \operatorname{ord}\left(\gamma_{n}\right)\right) \in \mathbb{N}^{n} .
$$

Note that $\phi_{*}(\gamma)=\phi_{*}(\tilde{\gamma})=\left(a_{1}, \ldots, a_{n}\right)=: a$ where $a \in \mathbb{N}^{n}$ is as in (17.3).

When $F$ is not flat, the composition map $\Phi_{*}: \Gamma^{*} \rightarrow \mathcal{F}_{F}^{C}$ can be defined by

$$
\Phi_{*}(\gamma)=\left(\kappa_{*} \circ \phi_{*}\right)(\gamma)
$$

from the sequence (17.2). For $\gamma \in \Gamma^{*}, \Phi_{*}(\gamma)$ is the bounded face defined by the hyperplane $H\left(\phi_{*}(\gamma), l_{*}\left(\phi_{*}(\gamma)\right)\right)$.

Lemma 7.1. Let $\gamma \in \Gamma^{*}$ and $l=l_{*}\left(\phi_{*}(\gamma)\right)$. If $F$ is not flat, then

$$
F \circ \gamma \equiv F_{\Phi_{*}(\gamma)} \circ \tilde{\gamma} \bmod \quad \widetilde{\mathcal{H}}_{l+1} \text {, }
$$

where $F_{\Phi_{*}(\gamma)} \circ \tilde{\gamma}$ belongs to $\mathcal{H}_{l}$. In particular, ord $(F \circ \gamma) \geq l_{*}\left(\phi_{*}(\gamma)\right)$ holds.

Proof. Let $F$ admit the Taylor series (1.4) at the origin. In this proof, we set

$$
\kappa=\Phi_{*}(\gamma), \quad a=\phi_{*}(\gamma), \quad l=l_{*}\left(\phi_{*}(\gamma)\right)
$$

for $\gamma \in \Gamma^{*}$. Taylor's formula implies that

$$
F(z, \bar{z})=F_{\kappa}(z, \bar{z})+R_{\kappa}(z, \bar{z}),
$$

where $R_{\kappa} \in C_{0}^{\infty}\left(\mathbb{C}^{n}\right)$ satisfies $\mathcal{N}_{+}\left(R_{\kappa}\right) \subset H_{+}(a, l+1)$. Substituting $z=\gamma(t)$ into (7.7), we have

$$
(F \circ \gamma)(t, \bar{t})=\left(F_{\kappa} \circ \gamma\right)(t, \bar{t})+\left(R_{\kappa} \circ \gamma\right)(t, \bar{t})
$$

First, let us consider the function

$$
\left(F_{\kappa} \circ \gamma\right)(t, \bar{t})=\sum_{\alpha+\beta \in \kappa} C_{\alpha \beta} \gamma(t)^{\alpha} \overline{\gamma(t)}^{\beta}
$$

where $C_{\alpha \beta}$ are as in (1.4). Note that each $\gamma_{j}$ can be expressed as $\gamma_{j}(t)=c_{j} t^{a_{j}}+r_{j}(t)$ where some $r_{j} \in \mathcal{O}_{0}(\mathbb{C})$ with $r_{j}(t)=O\left(|t|^{a_{j}+1}\right)$. Substituting these expressions into $\gamma(t)^{\alpha}$, we have

$$
\begin{aligned}
\gamma(t)^{\alpha} & =\prod_{j=1}^{n} \gamma_{j}(t)^{\alpha_{j}}=\prod_{j=1}^{n}\left(c_{j} t^{a_{j}}+r_{j}(t)\right)^{\alpha_{j}} \\
& =\prod_{j=1}^{n}\left(c_{j} t^{a_{j}}\right)^{\alpha_{j}}+R_{\alpha}(t)=c^{\alpha} t^{\langle a, \alpha\rangle}+R_{\alpha}(t),
\end{aligned}
$$


where $R_{\alpha} \in \mathcal{O}_{0}(\mathbb{C})$. Moreover, it is easy to see that $\operatorname{ord}\left(R_{\alpha}\right) \geq\langle a, \alpha\rangle+1$, that is, $R_{\alpha} \in \mathcal{H}_{\langle a, \alpha\rangle+1}$. Moreover, substituting (7.10) into (17.9), we have

$$
\begin{aligned}
\left(F_{\kappa} \circ \gamma\right)(t, \bar{t}) & =\sum_{\alpha+\beta \in \kappa} C_{\alpha \beta} c^{\alpha} \bar{c}^{\beta} t^{\langle a, \alpha\rangle} \bar{t}^{\langle a, \beta\rangle}+\tilde{R}_{\kappa}(t, \bar{t}) \\
& =\left(F_{\kappa} \circ \tilde{\gamma}\right)(t, \bar{t})+\tilde{R}_{\kappa}(t, \bar{t}),
\end{aligned}
$$

where $\tilde{R}_{\kappa} \in C_{0}^{\infty}(\mathbb{C})$. Moreover, it is easy to see that $\operatorname{ord}\left(\tilde{R}_{\kappa}\right) \geq\langle a, \alpha+\beta\rangle+1=l+1$, that is, $\tilde{R}_{\kappa} \in \widetilde{\mathcal{H}}_{l+1}$. On the other hand, since $\kappa$ is contained in the hyperplane $H(a, l)$, $F_{\kappa} \circ \tilde{\gamma}$ belongs to the class $\mathcal{H}_{l}$.

Next, let us consider the function $R_{\kappa} \circ \gamma$. Taylor's formula implies that

$$
R_{\kappa}(z, \bar{z})=\sum_{\alpha+\beta \in E} A_{\alpha \beta}(z, \bar{z}) z^{\alpha} \bar{z}^{\beta}
$$

where $E$ is a finite subset in $H_{+}(a, l+1) \cap \mathbb{N}_{0}^{n}$ and $A_{\alpha \beta} \in C_{0}^{\infty}\left(\mathbb{C}^{n}\right)$. Note that each $\gamma_{j}$ can be expressed as $\gamma_{j}(t)=d_{j}(t) t^{a_{j}}$ where $d_{j} \in \mathcal{O}_{0}(\mathbb{C})$ with $d_{j}(0)=c_{j}(\neq 0)$. Substituting $\gamma_{j}(t)=d_{j}(t) t^{a_{j}}$ for $j=1, \ldots, n$ into $\gamma(t)^{\alpha}$, we have

$$
\begin{aligned}
\gamma(t)^{\alpha} & =\prod_{j=1}^{n} \gamma_{j}(t)^{\alpha_{j}}=\prod_{j=1}^{n}\left(d_{j}(t) t^{a_{j}}\right)^{\alpha_{j}} \\
& =\left(\prod_{j=1}^{n} d_{j}(t)^{\alpha_{j}}\right) t^{\langle a, \alpha\rangle}=d(t)^{\alpha} t^{\langle a, \alpha\rangle} .
\end{aligned}
$$

Moreover, substituting (7.12) into (7.11), we have

$$
\begin{aligned}
\left(R_{\kappa} \circ \gamma\right)(t, \bar{t}) & =\sum_{\alpha+\beta \in E} B_{\alpha \beta}(t, \bar{t}) \gamma(t)^{\alpha} \overline{\gamma(t)}^{\beta} \\
& =\sum_{\alpha+\beta \in E} B_{\alpha \beta}(t, \bar{t}) d(t)^{\alpha} \overline{d(t)} t^{\langle a, \alpha\rangle} \bar{t}^{\langle a, \beta\rangle}
\end{aligned}
$$

where $B_{\alpha \beta}(t, \bar{t})=A_{\alpha \beta}(\gamma(t), \overline{\gamma(t)})$. Since $\langle a, \alpha+\beta\rangle \geq l+1$ if $\alpha+\beta \in H_{+}(a, l+1)$, (6.5) implies that if $\alpha+\beta \in E$, then

$$
\begin{aligned}
& \operatorname{ord}\left(B_{\alpha \beta}(t, \bar{t}) d(t)^{\alpha} \overline{d(t)}{ }^{\beta} t^{\langle a, \alpha\rangle} \bar{t}^{\langle a, \beta\rangle}\right) \\
= & \operatorname{ord}\left(B_{\alpha \beta}(t, \bar{t}) d(t)^{\alpha} \overline{d(t)}\right) \cdot \operatorname{ord}\left(t^{\langle a, \alpha\rangle}\right) \cdot \operatorname{ord}\left(\bar{t}^{\langle a, \beta\rangle}\right) \\
\geq & \langle a, \alpha\rangle+\langle a, \beta\rangle=\langle a, \alpha+\beta\rangle \geq l+1 .
\end{aligned}
$$

Thus, from (7.13), (7.14), we see that $R_{\kappa} \circ \gamma$ belongs to the class $\widetilde{\mathcal{H}}_{l+1}$. 
7.2. The restriction process. In order to treat the curves which are contained in some coordinate plane, we prepare many kinds of notation and symbols and must naturally generalize the maps constructed in the generic case. Hereafter in this section, $I$ is a nonempty subset of $\{1, \ldots, n\}$.

7.2.1. Notation and symbols. Let $K$ be one of the sets $\mathbb{N}, \mathbb{N}_{0}, \mathbb{R}, \mathbb{R}_{>}, \mathbb{R}_{\geq}, \mathbb{C}, \mathbb{C}^{*}$. We denote

$$
\widehat{K}:=K \cup\{\infty\}
$$

and

$$
K^{I}:=\left\{\left(X_{j}\right)_{j \in I}: X_{j} \in K\right\} .
$$

For $X=\left(X_{1}, \ldots, X_{n}\right) \in K^{n}$, we denote $X_{I}:=\left(X_{j}\right)_{j \in I} \in K^{I}$.

7.2.2. The maps $T_{I}^{(s)}, \iota_{I}^{(s)}, \pi_{I}$. Let $s=0$ or $\infty$. The map $T_{I}^{(s)}: K^{n} \rightarrow \widehat{K}^{n}$ is defined by

$$
\left(\hat{X}_{1}, \ldots, \hat{X}_{n}\right)=T_{I}^{(s)}\left(X_{1}, \ldots, X_{n}\right) \text { with } \hat{X}_{j}:= \begin{cases}X_{j} & \text { for } j \in I \\ s & \text { otherwise. }\end{cases}
$$

When $K=\mathbb{R}$ or $\mathbb{C}$, every coordinate plane in $K^{n}$ can be expressed by $T_{I}^{(0)}\left(K^{n}\right)$ for some $I \subset\{1, \ldots, n\}$, i.e.,

$$
T_{I}^{(0)}\left(K^{n}\right)=\left\{X \in K^{n}: X_{j}=0 \text { if } j \notin I\right\} .
$$

The map $\pi_{I}: K^{n} \rightarrow K^{I}$ is defined by

$$
\pi_{I}(X)=\pi_{I}\left(X_{1}, \ldots, X_{n}\right):=\left(X_{j}\right)_{j \in I}=X_{I} .
$$

Noticing $T_{I}^{(s)}(X) \in \widehat{K}^{n}$ depends only on $X_{I}$, we define an embedding map $\iota_{I}^{(s)}: K^{I} \rightarrow$ $\widehat{K}^{n}$ by

$$
\iota_{I}^{(s)}\left(X_{I}\right):=T_{I}^{(s)}(X) .
$$

It is easy to see that the following diagram commutes.

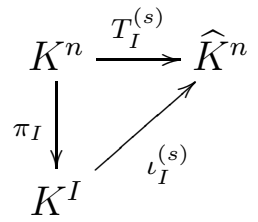

That is, $\iota_{I}^{(s)} \circ \pi_{I}=T_{I}^{(s)}$ holds. When $I=\{1, \ldots, n\}$, the maps $T_{I}^{(s)}, \iota_{I}^{(s)}, \pi_{I}$ are the identity map (i.e., $T_{I}^{(s)}(X)=\iota_{I}^{(s)}(X)=\pi_{I}(X)=X$ for $\left.X \in K^{n}\right)$. 
7.2.3. The function $F_{I}$. Let $z=\left(z_{1}, \ldots, z_{n}\right) \in \mathbb{C}^{n}$. Let $F_{I} \in C_{0}^{\infty}\left(\mathbb{C}^{I}\right)$ be the germ of the function of $z_{I}\left(=\left(z_{j}\right)_{j \in I} \in \mathbb{C}^{I}\right)$ defined by

$$
F_{I}\left(z_{I}\right):=\left(F \circ \iota_{I}^{(0)}\right)\left(z_{I}\right)
$$

When $I=\{1, \ldots, n\}, F_{I}\left(z_{I}\right)=F(z)$. Note that $F_{I}$ may be considered as the restriction of $F$ to the coordinate plane $T_{I}^{(0)}\left(\mathbb{C}^{n}\right)$ (see (17.18) ). The Newton polyhedron of $F_{I}$ is similarly defined, which is denoted by $\mathcal{N}_{+}\left(F_{I}\right)$. Note that $\mathcal{N}_{+}\left(F_{I}\right) \subset \mathbb{R}_{\geq}^{I}$.

7.2.4. A decomposition of $\widehat{\mathbb{N}}^{n}$. Let us consider the case where $K=\mathbb{N}$ and $s=\infty$ in Section 7.2.2. It is easy to see that

$$
\widehat{\mathbb{N}}^{n} \backslash\{\infty\}=\coprod_{I \subset\{1, \ldots, n\}} T_{I}^{(\infty)}\left(\mathbb{N}^{n}\right),
$$

where $\infty:=(\infty, \ldots, \infty)$ and the disjoint union is taken over all the nonempty subsets $I \subset\{1, \ldots, n\}$. Since the map $\iota_{I}^{(\infty)}$ is bijection, $T_{I}^{(\infty)}\left(\mathbb{N}^{n}\right)$ can be identified with $\mathbb{N}^{I}$. Therefore, $\widehat{\mathbb{N}}^{n} \backslash\{\infty\}$ is decomposed into the following disjoint union:

$$
\widehat{\mathbb{N}}^{n} \backslash\{\infty\}=\coprod_{I \subset\{1, \ldots, n\}} \mathbb{N}^{I} .
$$

For $\hat{a}=\left(\hat{a}_{1}, \ldots, \hat{a}_{n}\right) \in \widehat{\mathbb{N}}^{n} \backslash\{\infty\}$, let $I(\hat{a})$ be the subset of $\{1, \ldots, n\}$ defined by

$$
j \in I(\hat{a}) \Longleftrightarrow \hat{a}_{j}<\infty .
$$

When $\hat{a}_{j}<\infty$, we write $\hat{a}_{j}=a_{j}$. The map $\pi: \widehat{\mathbb{N}}^{n} \backslash\{\infty\} \rightarrow \coprod_{I \subset\{1, \ldots, n\}} \mathbb{N}^{I}$ is defined by

$$
\left.\pi(\hat{a}):=\left(\hat{a}_{j}\right)_{j \in I}\left(=\left(a_{j}\right)_{j \in I}=a_{I}\right) \quad \text { with } I=I(\hat{a}) \text { in (17.23) }\right) \text {. }
$$

For example, it is easy to see the following two-dimensional case.

$$
\begin{aligned}
\widehat{\mathbb{N}}^{2} & =\mathbb{N}^{2} \sqcup(\mathbb{N} \times\{\infty\}) \sqcup(\{\infty\} \times \mathbb{N}) \sqcup\{(\infty, \infty)\} \\
& =T_{\{1,2\}}^{(\infty)}\left(\mathbb{N}^{2}\right) \sqcup T_{\{1\}}^{(\infty)}\left(\mathbb{N}^{2}\right) \sqcup T_{\{2\}}^{(\infty)}\left(\mathbb{N}^{2}\right) \sqcup\{\infty\} \\
& =\mathbb{N}^{\{1,2\}} \sqcup \mathbb{N}^{\{1\}} \sqcup \mathbb{N}^{\{2\}} \sqcup\{\infty\} .
\end{aligned}
$$

7.2.5. The maps $l_{I}, \kappa_{I}, l$, $\kappa$. We define the maps $l_{I}: \mathbb{N}^{I} \rightarrow \widehat{\mathbb{N}}$ and $\kappa_{I}: \mathbb{N}^{I} \rightarrow \mathcal{F}_{F_{I}}$ as follows. When $F_{I}$ is not flat, set

$$
\begin{aligned}
& l_{I}\left(a_{I}\right):=\min \left\{\left\langle a_{I}, \xi_{I}\right\rangle_{I}: \xi_{I} \in \mathcal{N}_{+}\left(F_{I}\right)\right\}, \\
& \kappa_{I}\left(a_{I}\right):=\left\{\xi_{I} \in \mathcal{N}_{+}\left(F_{I}\right):\left\langle a_{I}, \xi_{I}\right\rangle_{I}=l_{I}\left(a_{I}\right)\right\} .
\end{aligned}
$$

When $F_{I}$ is flat, set $l_{I}\left(a_{I}\right):=\infty$ and $\kappa_{I}\left(a_{I}\right):=\emptyset$.

Now the maps: $l: \widehat{\mathbb{N}}^{n} \backslash\{\infty\} \rightarrow \widehat{\mathbb{N}}$ and $\kappa: \widehat{\mathbb{N}}^{n} \backslash\{\infty\} \rightarrow \mathcal{F}_{F}$ are defined by

$$
\begin{aligned}
& l(\hat{a}):=\left(l_{I} \circ \pi\right)(\hat{a}), \\
& \kappa(\hat{a}):=\left(\iota_{I}^{(0)} \circ \kappa_{I} \circ \pi\right)(\hat{a}),
\end{aligned}
$$


where $I=I(\hat{a})$ in (7.23),$\iota_{I}^{(0)}$ is as in (7.20) and $\pi$ is as in (7.24).

7.2.6. A decomposition of $\Gamma$. Let

$$
\Gamma^{I}:=\left\{\left(\gamma_{j}\right)_{j \in I}: \gamma_{j} \in \mathcal{O}_{0}(\mathbb{C}) \text { with } \gamma_{j}(0)=0 \text { and } \gamma_{j} \not \equiv 0 \text { for } j \in I\right\}
$$

In a similar fashion to the case of $\widehat{\mathbb{N}}^{n} \backslash\{\infty\}$ in $(7.22)$, the set $\Gamma$ can be decomposed into the following disjoint union:

$$
\Gamma=\coprod_{I \subset\{1, \ldots, n\}} \Gamma^{I}
$$

For $\gamma \in \Gamma$, let $I(\gamma)$ be a nonempty subset of $\{1, \ldots, n\}$ such that

$$
j \in I(\gamma) \Longleftrightarrow \gamma_{j} \not \equiv 0
$$

(Recall that $\Gamma$ does not contain the map $\gamma \equiv 0$.) The map $\tilde{\pi}: \Gamma \rightarrow \coprod_{I \subset\{1, \ldots, n\}} \Gamma^{I}$ is defined by

$$
\tilde{\pi}(\gamma)=\tilde{\pi}\left(\gamma_{1}, \ldots, \gamma_{n}\right):=\left(\gamma_{j}\right)_{j \in I} \text { with } I=I(\gamma)
$$

7.3. Asymptotics of $F_{I} \circ \gamma_{I}$. In this subsection, let $I \subset\{1, \ldots, n\}$ be arbitrarily given. For $z=\left(z_{1}, \ldots, z_{n}\right) \in \mathbb{C}^{n}$, let $F_{I}$ be as in (17.21). For $\left(a_{I}, l_{I}\right) \in \mathbb{N}_{0}^{I} \times \mathbb{N}_{0}$, define

$$
H_{I}\left(a_{I}, l_{I}\right):=\left\{\xi_{I} \in \mathbb{R}_{\geq}^{I}:\left\langle a_{I}, \xi_{I}\right\rangle_{I}=l_{I}\right\}
$$

Suppose that $F_{I}$ is not flat. Let us consider the sequence:

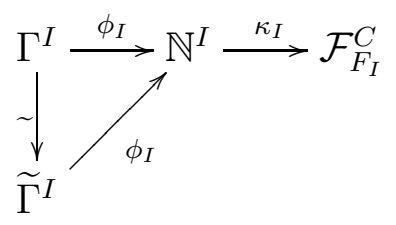

where $\Gamma^{I}$ is as in (7.27), $\mathcal{F}_{F_{I}}^{C}$ is the set of bounded faces of $\mathcal{N}_{+}\left(F_{I}\right)$ and

$$
\widetilde{\Gamma}^{I}:=\left\{\left(c_{j} t^{a_{j}}\right)_{j \in I}: c_{I} \in\left(\mathbb{C}^{*}\right)^{I}, a_{I} \in \mathbb{N}^{I}, t \in \mathbb{C}\right\},
$$

and the mappings $\sim, \phi_{I}$ are defined in a similar fashion to the generic case in (7.2).

In this setting, a similar argument to the generic case can be done in the space $\mathbb{C}^{I}$ or $\mathbb{R}_{\geq}^{I}$. When $F_{I}$ is not flat, the composition map $\Phi_{I}: \Gamma^{I} \rightarrow \mathcal{F}_{F_{I}}^{C}$ can be defined by

$$
\Phi_{I}\left(\gamma_{I}\right):=\left(\kappa_{I} \circ \phi_{I}\right)\left(\gamma_{I}\right)
$$

from the sequence (7.32). For $\gamma_{I} \in \Gamma^{I}, \Phi_{I}\left(\gamma_{I}\right)$ is the bounded face defined by the hyperplane $H_{I}\left(\phi_{I}\left(\gamma_{I}\right), l_{I}\left(\phi_{I}\left(\gamma_{I}\right)\right)\right)$, where $H_{I}$ is as in (7.31) and $l_{I}$ is as in (7.25). 
Lemma 7.2. Let $I$ be an nonempty subset of $\{1, \ldots, n\}$. Let $\gamma_{I} \in \Gamma^{I}$ and $l=l_{I}\left(\phi_{I}\left(\gamma_{I}\right)\right)$. If $F_{I}$ is not flat, then

$$
F_{I} \circ \gamma_{I} \equiv\left(F_{I}\right)_{\Phi_{I}\left(\gamma_{I}\right)} \circ \tilde{\gamma}_{I} \bmod \widetilde{\mathcal{H}}_{l+1},
$$

where $\left(F_{I}\right)_{\Phi_{I}\left(\gamma_{I}\right)} \circ \tilde{\gamma}_{I}$ belongs to $\mathcal{H}_{l}$. $\left(\left(F_{I}\right)_{\Phi_{I}\left(\gamma_{I}\right)}\right.$ means the $\Phi_{I}\left(\gamma_{I}\right)$-part of $F_{I}$. $)$ In particular, $\operatorname{ord}\left(F_{I} \circ \gamma_{I}\right) \geq l_{I}\left(\phi_{I}\left(\gamma_{I}\right)\right)$ holds.

Proof. The essential difference between Lemmas 7.1 and 7.2 is in the dimension. This lemma can be easily shown in a similar fashion to Lemma 7.1.

7.4. Asymptotics of $F \circ \gamma$ in the general case. Let us treat general complex curves in $\Gamma$.

The three maps $\phi: \Gamma \rightarrow \widehat{\mathbb{N}}^{n}, \Phi: \Gamma \rightarrow \mathcal{F}_{F}^{C}$ and $\sim: \Gamma \rightarrow \widetilde{\Gamma}$ are defined by

$$
\begin{aligned}
& \phi(\gamma):=\left(\iota_{I}^{(\infty)} \circ \phi_{I} \circ \tilde{\pi}\right)(\gamma), \\
& \Phi(\gamma):=\left(\iota_{I}^{(0)} \circ \Phi_{I} \circ \tilde{\pi}\right)(\gamma), \\
& \tilde{\gamma}:=\iota_{I}^{(0)}(\tilde{\tilde{\pi}(\gamma)}),
\end{aligned}
$$

for $\gamma \in \Gamma$, where $I=I(\gamma)$ (see (7.29) $)$ and $\iota_{I}^{(s)}(s=0$ or $\infty)$ is as in (7.20). When $\gamma \in \Gamma^{*}, \phi(\gamma)=\phi_{*}(\gamma), \Phi(\gamma)=\Phi_{*}(\gamma)$ and the last equation in (17.35) is trivial. It is easy to see that

$$
I(\gamma)=I(\phi(\gamma)), \quad l(\phi(\gamma))=\left(l_{I} \circ \phi_{I} \circ \tilde{\pi}\right)(\gamma)
$$

for $\gamma \in \Gamma$, where $I=I(\gamma)$ (see (7.23), (17.26) $)$ (17.29) $)$.

Theorem 7.3. Let $\gamma \in \Gamma$ and set $l=l(\phi(\gamma))$ and $I=I(\gamma)$. If $F_{I}$ is not flat, then

$$
F \circ \gamma \equiv F_{\Phi(\gamma)} \circ \tilde{\gamma} \bmod \quad \widetilde{\mathcal{H}}_{l+1},
$$

where $F_{\Phi(\gamma)} \circ \tilde{\gamma}$ belongs to $\mathcal{H}_{l}$. In particular, $\operatorname{ord}(F \circ \gamma) \geq l(\phi(\gamma))$ holds.

Proof. The definition of $I(\gamma)$ gives $F \circ \gamma=F_{I} \circ \gamma_{I}$. Lemma 7.2 implies

$$
F_{I} \circ \gamma_{I} \equiv\left(F_{I}\right)_{\Phi_{I}\left(\gamma_{I}\right)} \circ \tilde{\gamma}_{I} \bmod \widetilde{\mathcal{H}}_{l_{I}\left(\phi_{I}\left(\gamma_{I}\right)\right)+1}
$$

Noticing $\left(F_{I}\right)_{\Phi_{I}\left(\gamma_{I}\right)} \circ \tilde{\gamma}_{I}=F_{\Phi(\gamma)} \circ \tilde{\gamma}$ and (17.36) $)$, we can get the theorem.

Remark 7.4. The above theorem shows that $F_{\Phi(\gamma)}$ and $\tilde{\gamma}$ are appropriate truncations of $F$ and $\gamma$ in the computation of the order of contact of $F$ with $\gamma$. Indeed, if $F_{\Phi(\gamma)} \circ \tilde{\gamma} \not \equiv 0$, then $F$ and $\gamma$ in (1.12) can be replaced by $F_{\Phi(\gamma)}$ and $\tilde{\gamma}$. The details will be discussed in the next section. 
7.5. Nondegeneracy condition on $F_{I}$. Let $F_{I}$ be as in (7.21) and let $\kappa_{I}$ be a bounded face of $\mathcal{N}_{+}\left(F_{I}\right)$. Let $\widetilde{\Gamma}_{\kappa_{I}}^{*}:=\left\{\left(c_{j} t^{a_{j}}\right)_{j \in I}: a_{I}\right.$ determines $\left.\kappa_{I}\right\}$, where $c_{I}=\left(c_{j}\right)_{j \in I} \in\left(\mathbb{C}^{*}\right)^{I}$ and $a_{I}=\left(a_{j}\right)_{j \in I} \in \mathbb{N}^{I}$.

Definition 7.5. Let $\kappa_{I}$ be a bounded face of $\mathcal{N}_{+}\left(F_{I}\right)$. The $\kappa_{I}$-part $\left(F_{I}\right)_{\kappa_{I}}$ of $F_{I}$ is said to be nondegenerate if

$$
\left(F_{I}\right)_{\kappa_{I}} \circ \gamma_{I} \not \equiv 0 \quad \text { for any } \gamma_{I} \in \widetilde{\Gamma}_{\kappa_{I}}^{*} .
$$

Now, let us consider the case where a bounded face $\kappa$ of $\mathcal{N}_{+}(F)$ is contained in some coordinate plane. Let $I(\kappa)$ be the minimal subset $I$ of $\{1, \ldots, n\}$ such that $\kappa \subset T_{I}^{(0)}\left(\mathbb{R}^{n}\right)$ (see (7.18)). Hereafter we denote $I:=I(\kappa)$ and $\kappa_{I}:=\pi_{I}(\kappa) \subset\left(\mathbb{R}_{\geq}\right)^{I}$. Note that $\kappa$ can be identified with $\kappa_{I}$ and that $\kappa=\iota_{I}^{(0)}\left(\kappa_{I}\right)$.

Lemma 7.6. The following two conditions are equivalent.

(i) $F_{\kappa}$ is nondegenerate;

(ii) $\left(F_{I}\right)_{\kappa_{I}}$ is nondegenerate.

Proof. ((i) $\Longrightarrow$ (ii).) $\quad$ Let $\gamma_{I}=\left(\gamma_{j}\right)_{j \in I} \in \widetilde{\Gamma}_{\kappa_{I}}^{*}$ be arbitrarily given. Let $\check{\gamma}=\left(\check{\gamma}_{1}, \ldots, \check{\gamma}_{n}\right) \in$ $\Gamma$ be defined by $\check{\gamma}_{j}=\gamma_{j}$ if $j \in I$ and $\check{\gamma}_{j}(t)=t^{m}$ if $j \notin I$ with $m \in \mathbb{N}$. We can choose $m$ such that $\check{\gamma}$ belongs to $\widetilde{\Gamma}_{\kappa_{I}}^{*}$. Note that $\gamma_{I}=\pi_{I} \circ \check{\gamma}$ and $\left(F_{I}\right)_{\kappa_{I}} \circ \pi_{I}=F_{\kappa}$. Since $\left(F_{I}\right)_{\kappa_{I}} \circ \gamma_{I}=\left(F_{I}\right)_{\kappa_{I}} \circ \pi_{I} \circ \check{\gamma}=F_{\kappa} \circ \check{\gamma}$, (i) implies $\left(F_{I}\right)_{\kappa_{I}} \circ \gamma_{I} \not \equiv 0$.

$\left((\mathrm{ii}) \Longrightarrow(\mathrm{i})\right.$.) $\quad$ Let $\gamma=\left(\gamma_{1} \ldots, \gamma_{n}\right) \in \widetilde{\Gamma}_{\kappa}^{*}$ be arbitrarily given. Let $\gamma_{I}=\left(\gamma_{j}\right)_{j \in I} \in \Gamma^{I}$. Then $\gamma_{I}$ belongs to $\widetilde{\Gamma}_{\kappa_{I}}^{*}$. Note that $\iota_{I}^{(0)} \circ \pi_{I}$ is the identity map. Since $F_{\kappa} \circ \gamma=$ $F_{\kappa} \circ \iota_{I}^{(0)} \circ \pi_{I} \circ \gamma=\left(F_{I}\right)_{\kappa_{I}} \circ \gamma_{I}$, (ii) implies $F_{\kappa} \circ \gamma \not \equiv 0$.

\section{ORDER OF CONTACT FOR SMOOTH FUNCTIONS}

Let $F \in C_{0}^{\infty}\left(\mathbb{C}^{n}\right)$ with $F(0,0)=0$ and let $\Gamma$ be as in (3.1).

8.1. Newton distance and $n$-tuple of numbers. In this subsection, let $\hat{a} \in \widehat{\mathbb{N}}^{n} \backslash$ $\{\infty\}$ and set $I=I(\hat{a})($ see (7.23) $)$. Note that $\hat{a}_{I}=\left(\hat{a}_{j}\right)_{j \in I}=\left(a_{j}\right)_{j \in I}=a_{I} \in \mathbb{N}^{I}$.

The Newton distance of $F$ in the direction $\hat{a}$ is defined by

$$
d(F, \hat{a}):=\frac{l(\hat{a})}{\min \left\{a_{j}: j=1, \ldots, n\right\}}\left(=\frac{l_{I}\left(a_{I}\right)}{\min \left\{a_{j}: j \in I\right\}}\right),
$$

where $l, l_{I}$ are as in (7.26), (7.25). We define the $n$-tuple of numbers:

$$
\rho(F, \hat{a}):=\left(\rho_{1}(F, \hat{a}), \ldots, \rho_{n}(F, \hat{a})\right) \in \widehat{\mathbb{N}}_{0}^{n}
$$

as follows.

(i) When $F_{I}$ is not flat and $j \in I$, let $\rho_{j}(F, \hat{a})$ be the coordinate of the intersection of the hyperplane $H_{I}\left(a_{I}, l_{I}\left(a_{I}\right)\right)$ (see (7.31) $)$ in $\mathbb{R}_{\geq}^{I}$ with the $\xi_{j}$-axis;

(ii) When $F_{I}$ is flat and $j \in I$, we set $\rho_{j}(F, \hat{a})=\infty$; 
(iii) If $j \notin I$, then we set $\rho_{j}(F, \hat{a})=0$.

When $F_{I}$ is not flat, we similarly define $\rho\left(F_{I}, a_{I}\right)=\left(\rho_{j}\left(F_{I}, a_{I}\right)\right)_{j \in I} \in \mathbb{N}^{I}$ as in (i).

The following lemma shows the relationship between $\rho(F, \hat{a})$ and $\rho(F)$ (see (2.9) $)$.

Lemma 8.1. $\rho_{j}(F, \hat{a}) \leq \rho_{j}(F)$ for $\hat{a} \in \widehat{\mathbb{N}}^{n} \backslash\{\infty\}, j=1, \ldots, n$.

Proof. From the geometrical relationship: $\mathcal{N}_{+}\left(F_{I}\right) \subset H_{+}\left(a_{I}, l_{I}\left(a_{I}\right)\right)$, the definitions of $\rho(F)$ and $\rho(F, \hat{a})$ easily imply the inequalities in the lemma.

By considering the geometrical meaning of $l(\hat{a})$, the quantity $d(F, \hat{a})$ stands for some kind of "distance" from the origin to $\mathcal{N}_{+}(F)$ in the direction $\hat{a}$. The following lemma implies that this quantity can be expressed by using $\rho(F, \hat{a})$. Since $\rho(F, \hat{a})$ can be more directly computed, it will be convenient to see the value of $d(F, \hat{a})$.

Lemma 8.2. $d(F, \hat{a})=\max \left\{\rho_{j}(F, \hat{a}): j=1, \ldots, n\right\}$ for $\hat{a} \in \widehat{\mathbb{N}}^{n} \backslash\{\infty\}$.

Proof. Recalling that the hyperplane $H\left(\phi_{I}\left(\gamma_{I}\right), a_{I}\right)$ is defined by the equation:

$$
\left\langle a_{I}, \xi_{I}\right\rangle_{I}=\sum_{j \in I} a_{j} \cdot \xi_{j}=l_{I}\left(a_{I}\right),
$$

we can see $\rho_{j}(F, \hat{a})=l_{I}\left(a_{I}\right) \cdot a_{j}^{-1}$ for $j \in I$. By using these equalities,

$$
\begin{aligned}
& \max \left\{\rho_{j}(F, \hat{a}): j=1, \ldots, n\right\}=\max \left\{\rho_{j}(F, \hat{a}): j \in I\right\} \\
& \quad=\max \left\{\frac{l_{I}\left(a_{I}\right)}{a_{j}}: j \in I\right\}=\frac{l_{I}\left(a_{I}\right)}{\min \left\{a_{j}: j \in I\right\}}=d(F, \hat{a}) .
\end{aligned}
$$

8.2. Order of contact and the Newton distance. Let us consider the relationship between the order of contact and the Newton distance. In this subsection, let $\gamma \in \Gamma$ be arbitrarily given and set $I=I(\gamma)$ (see (17.29) ).

Proposition 8.3. (i) $\operatorname{ord}(F \circ \gamma) \geq l(\phi(\gamma))$, (ii) $O(F, \gamma) \geq d(F, \phi(\gamma))$.

Proof. When $F_{I}$ is flat, we see that $\operatorname{ord}(F \circ \gamma)=\operatorname{ord}\left(F_{I} \circ \gamma_{I}\right)=\infty$, which imply the estimates in (i), (ii). Therefore, it suffices to consider the case when $F_{I}$ is not flat.

The estimate in (i) has been stated in Theorem 7.3. By noticing that (i) is equivalent to $\operatorname{ord}\left(F_{I} \circ \gamma_{I}\right) \geq l_{I}\left(\phi_{I}\left(\gamma_{I}\right)\right)$, (8.1) gives

$$
O(F, \gamma)=O\left(F_{I}, \gamma_{I}\right) \geq \frac{l_{I}\left(\phi_{I}\left(\gamma_{I}\right)\right)}{\min \left\{\operatorname{ord}\left(\gamma_{j}\right): j \in I\right\}}=d(F, \phi(\gamma)),
$$

which is the estimate in (ii).

The following theorem shows that the order of contact of $\gamma$ with $F$ exactly equals the distance of $F$ in the direction $\phi(\gamma)$ under the nondegeneracy assumption. 
Theorem 8.4. If $F_{\Phi(\gamma)}$ is nondegenerate, then we have

$$
\text { (i) } \operatorname{ord}(F \circ \gamma)=l(\phi(\gamma)), \quad \text { (ii) } O(F, \gamma)=d(F, \phi(\gamma)) \text {. }
$$

In particular, if $F$ is nondegenerate, then the equalities in (i), (ii) hold for any $\gamma \in \Gamma$.

Proof. From the proof of Proposition 8.3, it suffices to show the following lemma.

Lemma 8.5. If $F_{\Phi(\gamma)}$ is nondegenerate, then $\operatorname{ord}\left(F_{I} \circ \gamma_{I}\right)=l_{I}\left(\phi_{I}\left(\gamma_{I}\right)\right)$ with $I=I(\gamma)$.

Proof. Note that $\Phi(\gamma)=\iota_{I}^{(0)}\left(\Phi_{I}\left(\gamma_{I}\right)\right)$ from $I=I(\gamma)$. First, let us consider the case when $F_{I}$ is not flat. Since the nondegeneracy condition of $F_{\Phi(\gamma)}$ implies that of $\left(F_{I}\right)_{\Phi_{I}\left(\gamma_{I}\right)}$ from Lemma 7.6, we have $\operatorname{ord}\left(\left(F_{I}\right)_{\Phi_{I}\left(\gamma_{I}\right)} \circ \tilde{\gamma}_{I}\right)=l_{I}\left(\phi_{I}\left(\gamma_{I}\right)\right)$ from the equivalence (6.4). Therefore, it follows from the asymptotics in Lemma 7.2 that

$$
\operatorname{ord}\left(F_{I} \circ \gamma_{I}\right)=\operatorname{ord}\left(\left(F_{I}\right)_{\Phi_{I}\left(\gamma_{I}\right)} \circ \tilde{\gamma}_{I}\right)=l_{I}\left(\phi_{I}\left(\gamma_{I}\right)\right) \text {. }
$$

Next, let us consider the case when $F_{I}$ is flat. Since $F_{I} \circ \gamma_{I}$ is also flat, we have $\operatorname{ord}\left(F_{I} \circ \gamma_{I}\right)=\infty$. Recall that $l_{I}\left(\gamma_{I}\right)=\infty$, then the equality in the lemma can be obtained.

\section{Proof of Theorem 1.3}

We are now in a position to prove Theorem 1.3.

Let $(z)$ be a canonical coordinate for $M$ at $p$. Recall that $\rho_{j}(M, p ;(z))=\rho_{j}(r)$ for $j=1, \ldots, n$ (see (2.9),$(2.11)$ ). From Theorem 1.1, it suffices to show that $\Delta_{1}(M, p) \leq$ $\rho_{1}(r)$.

Since the defining function $r$ for $M$ has the nondegeneracy condition on $(z)$, Theorem 8.4 and Lemma 8.1 imply that the order of contact of $\gamma \in \Gamma$ with $M$ can be estimated as follows.

$$
\begin{aligned}
O(r, \gamma) & =d(r, \phi(\gamma))=\max \left\{\rho_{j}(r, \phi(\gamma)): j=1, \ldots, n\right\} \\
& \leq \max \left\{\rho_{j}(r): j=1, \ldots, n\right\}=\rho_{1}(r),
\end{aligned}
$$

for any $\gamma \in \Gamma$. This estimate gives $\Delta_{1}(M, p) \leq \rho_{1}(r)$.

\section{ON NORMALIZED COORDINATES}

It follows from Theorem 1.3 that when a hypersurface admits a canonical coordinate, important properties of the regular and singular types can be understood. On the other hand, it is another serious issue to determine the existence of canonical coordinates for a given hypersurface and, if they exist, to actually construct these coordinates. The rest of this paper is to devote this issue. In this section, we prepare more convenient coordinates for this investigation in special cases treated in Sections 11-15.

Let $M$ be a real hypersurface in $\mathbb{C}^{n+1}$ and let $p$ lie on $M$. 
10.1. Normalization of coordinates. It is not always easy to check the nondegeneracy condition. This difficulty is sometimes caused by the existence of pure terms in the principal part of a defining function. In order to avoid these situation, we prepare a convenient coordinate on which a local defining function is of more useful form.

Lemma 10.1. For any $N \in \mathbb{N}$, there exists a holomorphic coordinate $(z, w):=$ $\left(z_{1}, \ldots, z_{n}, w\right)$ at $p$ on which a defining function $r$ for $M$ is expressed near the origin as in the following form:

$$
r(z, w, \bar{z}, \bar{w})=\operatorname{Re}(w)+F(z, \bar{z})+R_{1}(z, \bar{z}) \cdot \operatorname{Im}(w)+R_{2}(z, w, \bar{z}, \bar{w}),
$$

where

(i) $F \in C_{0}^{\infty}\left(\mathbb{C}^{n}\right)$ satisfies that

(a) $F(0,0)=0$ and $\nabla F(0,0)=0$;

(b) If $\mathcal{N}_{+}(F) \cap\{\xi:|\xi| \leq N\} \neq \emptyset$, then $j_{N} F$ (see 1.14) contains no pure terms;

(c) $\rho(F)$ satisfies $\rho_{j}(F)=\rho_{j}(M, p ;(z, w)) \geq 2$ for $j=1, \ldots, n$ (see (2.9), (2.11));

(ii) $R_{1} \in C_{0}^{\infty}\left(\mathbb{C}^{n}\right)$ and $R_{2} \in C_{0}^{\infty}\left(\mathbb{C}^{n+1}\right)$. Moreover, $R_{2}$ satisfies that $\left|R_{2}(z, w, \bar{z}, \bar{w})\right| \leq$ $C|w|^{2}$ near $(z, w)=0$ where $C$ is a positive constant independent of $(z, w)$.

Proof. Taylor's formula easily implies all the above properties except (i-b). A simple change of coordinates gives the property (i-b).

We call the above coordinate $(z, w)$ a normalized coordinate for $M$ at $p$ with $N \in \mathbb{N}$. Of course, there are many such coordinates for a given $M$ and $N \in \mathbb{N}$. Hereafter, normalized coordinates will be often used and the value of $N$ is, if possible, assumed to be so large that the principal part of $F$ contains no pure term without any mentioning (see (1.15) for the definition of the principal part). It can be easily seen that the above integer $N$ always exists when $\rho_{1}(M, p)<\infty$. On the other hand, in the case of the real hypersurface $M$ with $\rho_{1}(M, 0)=\infty$ in [3], [13] explained in Section 4.1, for every integer $N$ no matter how large, a pure term necessarily appears in the principal part of $F$ on normalized coordinates $(z, w)$ with $N$.

Remark 10.2. In the condition (ii) in Lemma 10.1, $\left|R_{2}\right| \leq C_{2}|w|^{2}$ can be modified as $\left|R_{2}\right| \leq C_{2}|\operatorname{Im}(w)|^{2}$. But, the estimate in (ii) is sufficient for our purpose and is more convenient for the application (see Section 13).

10.2. An equivalence condition for canonical coordinates. After being normalized as in Lemma 10.1, it is relatively easy to check the canonical conditions.

Proposition 10.3. Let $(z, w)$ be a normalized coordinate for $M$ at $p$ in Lemma 10.1. If the principal part $F_{0}$ of $F$ contains no pure terms, the following two conditions are equivalent.

(i) A coordinate $(z, w)$ is canonical for $M$; 
(ii) $F$ is nondegenerate.

This proposition easily follows from the following lemma.

Lemma 10.4. Let $r, F$ be as in Lemma 10.1. If the principal part $F_{0}$ of $F$ contains no pure terms, then the following conditions are equivalent.

(i) $F$ is nondegenerate;

(ii) $r$ is nondegenerate.

Proof. The implication: (ii) $\Longrightarrow$ (i) is obvious. In order to show the implication: (i) $\Longrightarrow$ (ii), it suffices to show that $G_{\kappa}(z, w, \bar{z}, \bar{w})=2 \operatorname{Re}(w)+F_{\kappa}(z, \bar{z})$ is always nondegenerate for every bounded face $\kappa$ of $\mathcal{N}_{+}(F)$. We assume that $G_{\kappa}$ is not nondegenerate. Then, there exists a curve $\tilde{\gamma}=\left(\gamma, \gamma_{n+1}\right):(\mathbb{C}, 0) \rightarrow\left(\mathbb{C}^{n+1}, 0\right)$ contained in $\left(\mathbb{C}^{*}\right)^{n+1} \cup\{0\}$ such that $G_{\kappa} \circ \tilde{\gamma} \equiv 0$. From this equation, we see $2 \operatorname{Re}\left(\gamma_{n+1}(t)\right)=-F_{\kappa}(\gamma(t), \overline{\gamma(t)})$. Now, $2 \operatorname{Re}\left(\gamma_{n+1}(t)\right)$ is harmonic, but $F_{\kappa} \circ \gamma(\not \equiv 0)$ is not so, which is a contradiction.

Remark 10.5. In the above lemma, the assumption that the principal part $F_{0}$ of $F$ contains no pure terms is necessary. Indeed, $F(z, \bar{z})=\left|z_{1}-z_{2}\right|^{2}+2 \operatorname{Re}\left(z_{1} z_{2}\right)$ is nondegenerate, but $r(z, w, \bar{z}, \bar{w})=2 \operatorname{Re}(w)+F(z, \bar{z})$ is degenerate (consider the curve: $\left.\gamma(t)=\left(t, t,-t^{2}\right)\right)$.

10.3. On canonical normalized coordinates. On the coordinates in Lemma 10.1, the regular and singular types can be determined by the information of $F$ only under nondegeneracy conditions. More exactly, these types are equal to the order of contact with the function $F$. Let $\Gamma$ be as in (3.1).

Proposition 10.6. Let $(z, w)$ be a normalized coordinate for $M$ at $p$ in Lemma 10.1. If the principal part $F_{0}$ of $F$ contains no pure terms and $F$ is nondegenerate, then

$$
\Delta_{1}(M, p)=\Delta_{1}^{\mathrm{reg}}(M, p)=\sup _{\gamma \in \Gamma} O(F, \gamma)=\rho_{1}(F)
$$

In particular, $M$ is of finite type at $p$ if and only if $F$ is convenient.

Proof. Since $F$ is nondegenerate, Theorem 8.4 and Lemmas 8.1 and 8.2 imply

$$
\begin{aligned}
O(F, \gamma) & =d(F, \phi(\gamma))=\max \left\{\rho_{j}(F, \phi(\gamma)): j=1, \ldots, n\right\} \\
& \leq \max \left\{\rho_{j}(F): j=1, \ldots, n\right\}=\rho_{1}(F) .
\end{aligned}
$$

Note that there exists a holomorphic mapping $\gamma$ attaining the equality in (10.2). Since $\rho_{j}(F)=\rho_{j}(r)$ for $j=1, \ldots, n$ and $\rho_{n+1}(r)=\rho_{n+1}(M, p ;(z, w))=1$, (10.2) and Theorem 1.3 with Lemma 10.4 imply

$$
\sup _{\gamma \in \Gamma} O(F, \gamma)=\rho_{1}(F)=\rho_{1}(r)=\rho_{1}(M, p ;(z, w))=\Delta_{1}(M, p) .
$$




\section{Pseudoconvexity}

Let $M$ be a real hypersurface in $\mathbb{C}^{n+1}$ and let $p$ lie on $M$. In the function theory of several complex variables, the case where $M$ is the boundary of a pseudoconvex domain is essentially important. Let us investigate plurisubharmonicity and pseudoconvexity from the viewpoint of the notion of Newton polyhedra. We say that a real hypersurface $M=\partial \Omega$ is pseudoconvex at $p$ if $\Omega$ is pseudoconvex at $p$.

Let $F \in C_{0}^{\infty}\left(\mathbb{C}^{n}\right)$ with $F(0,0)=0$ and let $\Gamma$ be as in (3.1).

11.1. Plurisubharmonicity. The following lemma is valuable and has also been seen in [14]. Its proof is easy, so it is omitted.

Lemma 11.1. If $F \in C_{0}^{\infty}\left(\mathbb{C}^{n}\right)$ is plurisubharmonic, then so is the $\kappa$-part $F_{\kappa}$ of $F$ for every bounded face $\kappa$.

We remark that the converse of the above lemma is not true. For example, consider the function $F(z, \bar{z})=\left|z_{1}-z_{2}\right|^{2}-\left|z_{2}\right|^{4}$.

11.2. Property PS. The notion of property PS was introduced by D'Angelo [7], [9], which is a certain positivity condition, more general than plurisubharmonicity or pseudoconvexity. It is known that this property is often sufficient for the investigation of order of contacts to see their important properties.

Definition 11.2. We say that $F \in C_{0}^{\infty}\left(\mathbb{C}^{n}\right)$ satisfies property $\mathbf{P S}$ at 0 if, for any $\gamma \in \Gamma$ for which $\operatorname{ord}(F \circ \gamma)<\infty$,

(i) $\operatorname{ord}(F \circ \gamma)$ is even, say $2 m$;

(ii) $(d / d t)^{m}(d / d \bar{t})^{m}(F \circ \gamma)(0,0)>0$.

From the definition, if $F$ is plurisubharmonic and the Taylor series of $F$ contains no pure terms, then $F$ satisfies property PS at 0 (see [7], [9]). Recall the principal part $F_{0}$ of $F$ :

$$
F_{0}(z, \bar{z})=\sum_{\alpha+\beta \in \mathcal{N}(F)} C_{\alpha \beta} z^{\alpha} \bar{z}^{\beta},
$$

where $\mathcal{N}(F)$ is the Newton diagram of $F$ (i.e., the union of all bounded faces of $\mathcal{N}_{+}(F)$ ).

Proposition 11.3. Suppose that the principal part $F_{0}$ of $F$ has no pure term. If $F$ satisfies property $\mathbf{P S}$, then the $\mathbf{v}$-part $F_{\mathbf{v}}$ of $F$ contains a term of the form $c\left|z_{1}\right|^{v_{1}} \cdots\left|z_{n}\right|^{v_{n}}$, with some $c>0$, for any vertex $\mathbf{v}=\left(v_{1}, \ldots, v_{n}\right)$ of $\mathcal{N}_{+}(F)$.

Remark 11.4. The converse of the above proposition does not always hold. Indeed, consider the case when $F(z, \bar{z})=\left|z_{1}-z_{2}\right|^{2}+\operatorname{Re}\left(z_{1}^{2} \bar{z}_{2}^{3}\right)$ and $\gamma(t)=(t, t)$.

Proof. Let $F$ admit the Taylor series (1.4) at the origin.

First, we consider the generic case: the vertex $\mathbf{v}=\left(v_{1}, \ldots, v_{n}\right)$ is away from any coordinate planes, i.e., $\mathbf{v} \in \mathbb{N}^{n}\left(\Leftrightarrow v_{j}>0\right.$ for all $\left.j\right)$. Then there exists the set of valid pairs $\left\{\left(a^{(j)}, l^{(j)}\right): j=1, \ldots, n\right\}$ for $\mathcal{N}_{+}(F)$ such that 
(a) The set of vectors: $\left\{a^{(j)}: j=1, \ldots, n\right\}$ is linearly independent;

(b) $H\left(a^{(j)}, l^{(j)}\right) \cap \mathcal{N}_{+}(F)=\mathbf{v}$.

Let $\gamma^{(j)}(t):=\left(t^{a_{1}^{(j)}}, \ldots, t^{a_{n}^{(j)}}\right)$. Since $\Phi\left(\gamma^{(j)}\right)=\mathbf{v}$ from the above $(\mathrm{b}), \gamma^{(j)}$ belongs to $\widetilde{\Gamma}_{\mathbf{v}}^{*}($ see $(3.1))$. Lemma 7.1 implies $F\left(\gamma^{(j)}(t), \overline{\gamma^{(j)}(t)}\right)=F_{\mathbf{v}}\left(\gamma^{(j)}(t), \overline{\gamma^{(j)}(t)}\right)+o\left(t^{l^{(j)}}\right)$. Substituting $\gamma^{(j)}(t)=\left(t^{a_{1}^{(j)}}, \ldots, t^{a_{n}^{(j)}}\right)$ into the $\mathbf{v}$-part $F_{\mathbf{v}}$ of $F$, we have

$$
F_{\mathbf{v}}\left(\gamma^{(j)}(t), \overline{\gamma^{(j)}(t)}\right)=\sum_{\alpha+\beta=\mathbf{v}} C_{\alpha \beta} t^{\left\langle a^{(j)}, \alpha\right\rangle} \bar{t}^{\left\langle a^{(j)}, \beta\right\rangle} .
$$

Therefore, property PS gives that, for each $j=1, \ldots, n$, there exist $\alpha^{(j)}, \beta^{(j)} \in \mathbb{N}_{0}^{n}$ such that

$$
\left\langle a^{(j)}, \alpha^{(j)}\right\rangle=\left\langle a^{(j)}, \beta^{(j)}\right\rangle, \quad \alpha^{(j)}+\beta^{(j)}=\mathbf{v}, \quad C_{\alpha^{(j)} \beta^{(j)}}>0 .
$$

Since the linearly independence condition (a) implies

$$
\bigcap_{j=1}^{n}\left\{\xi \in \mathbb{N}_{0}^{n}:\left\langle a^{(j)}, \xi\right\rangle=0\right\}=\{0\},
$$

we can see that $\alpha^{(j)}=\beta^{(j)}$ and $\alpha^{(j)}+\beta^{(j)}=\mathbf{v}$ for $j=1, \ldots, n$, which imply

$$
\alpha^{(j)}=\beta^{(j)}=\frac{1}{2} \mathbf{v} \text { for } j=1, \ldots, n \text {. }
$$

Next, we consider the general vertex $\mathbf{v}=\left(v_{1}, \ldots, v_{n}\right)$ of $\mathcal{N}_{+}(F)$, i.e., $\mathbf{v} \in \mathbb{N}_{0}^{n}$. Let $I:=\left\{j: v_{j} \neq 0\right\} \subset\{1, \ldots, n\}$. Let $F_{I}$ be as in (7.21). Since an essential difference between $F_{I}$ and the above generic case of $F$ is only in the dimension, the general case can be shown in a similar fashion to the generic case.

From Proposition 11.3, we can easily see the following.

Corollary 11.5. Suppose that $F$ satisfies property PS at 0 . Then every component of the coordinate of the vertices of $\mathcal{N}_{+}(F)$ is even.

Let us recall the definition of property PS in the case of real hypersurfaces in $\mathbb{C}^{n}$.

Definition 11.6. Let $M$ be a real hypersurface and let $p$ lie on $M$. Let us consider a normalized coordinate for $M$ at $p$ with large $N$ and a local defining function $r$ for $M$ near $p$ as in (10.1) in Lemma 10.1. We say that $M$ satisfies property PS at $p$ if there exists a $N_{0} \in \mathbb{N}$ such that if $N \geq N_{0}$ then $F$ in (10.1) satisfies property PS at 0 .

It is known that every pseudoconvex hypersurface satisfies property PS. Refer to [7], 9] for more detailed explanation of property PS. 


\section{The Semiregular ( $h$-EXtendible) CASE}

The semiregular (or $h$-extendible) class was independently introduced in [12], [39]. This class contains many kinds of finite type pseudoconvex domains: for example, strictly pseudoconvex domains in $\mathbb{C}^{n}$, pseudoconvex domains in $\mathbb{C}^{n}$ whose boundaries have at most one zero eigenvalue in the Levi form (in particular, the two-dimensional case), decoupled domains and convex domains in $\mathbb{C}^{n}$, etc.

This class can be variously characterized. The following are important.

- The equality holds in the inequality between the components of the Catlin multitype and the D'Angelo variety type: $m_{n-q+1}=\Delta_{q}<\infty$ for $q=1, \ldots, n$ (the definition of semiregular in [12]);

- They can be approximated by quasihomogeneous model (see Lemma 12.2 below);

- There exists a bumping function satisfying quasihomogenous property (the definition of $h$-extendible in [39]).

12.1. Quasihomogeneous model case. In the quasihomogeneous model, we show that the nondegeneracy condition exactly corresponds to the finite type condition.

Let $P$ be a real-valued polynomial of $z \in \mathbb{C}^{n}$ having the property:

$$
P\left(r^{1 / m} \bullet z, \overline{r^{1 / m} \bullet z}\right)=r P(z, \bar{z}) \quad \text { for all } r>0, z \in \mathbb{C}^{n},
$$

where $m=\left(m_{1}, \ldots, m_{n}\right) \in \mathbb{N}^{n}$ with $m_{j} \geq 2$ and

$$
r^{1 / m} \bullet z:=\left(r^{1 / m_{1}} z_{1}, \ldots, r^{1 / m_{n}} z_{n}\right) .
$$

Let us consider the real hypersurface:

$$
M_{P}:=\left\{(z, w) \in \mathbb{C}^{n+1}: r_{P}(z, w, \bar{z}, \bar{w}):=\operatorname{Re}(w)+P(z, \bar{z})=0\right\} .
$$

Note that the property (12.1) is equivalent to the condition that the Newton diagram $\mathcal{N}(P)$ consists of only one facet defined by $\left\{\xi \in \mathbb{R}_{\geq}^{n}: \sum_{j=1}^{n} \xi_{j} / m_{j}=1\right\}$.

Lemma 12.1. As for the hypersurface $M_{P}$, the following two conditions are equivalent.

(i) $P$ is convenient and nondegenerate;

(ii) The origin is of finite type.

Proof. Easy.

12.2. Existence of canonical coordinates. Let $\Omega$ be a domain with smooth boundary and let $p$ lie on $\partial \Omega$. As mentioned in the beginning of this section, the following is one of the characterizations of semiregular class.

Lemma 12.2 ([12] Theorem 1.9, [39]). The following two conditions are equivalent.

(1) $\partial \Omega$ is of semiregular type (h-extendible) at $p$; 
(2) There exists a normalized coordinate $(z, w)$ around $p$ on which a defining function for $\Omega$ near the origin can be expressed as in (10.1) in Lemma 10.1. Here $R_{1}, R_{2}$ satisfy the condition (ii) in Lemma 10.1 and, moreover, the principal part $F_{0}$ (see (11.1)) of $F$ satisfies the following conditions:

(a) $F_{0}$ contains no pure terms;

(b) $F_{0}$ is a plurisubharmonic polynomial;

(c) $F_{0}$ satisfies the equation:

$$
\begin{aligned}
& \quad F_{0}\left(r^{1 / m} \bullet z, \overline{r^{1 / m} \bullet z}\right)=r F_{0}(z, \bar{z}) \text { for all } r>0, z \in \mathbb{C}^{n}, \\
& \text { where } r^{1 / m} \bullet z \text { is as in (12.2) and } m=\left(m_{1}, \ldots, m_{n}\right) \in \mathbb{N}^{n} \text { with } m_{1} \geq \\
& \cdots \geq m_{n} \geq 2 \text {; } \\
& \text { (d) As for the domain } \Omega_{0}:=\left\{(z, w): \operatorname{Re}(w)+F_{0}(z, \bar{z})<0\right\} \text {, the origin is of } \\
& \text { finite type. }
\end{aligned}
$$

From the above characterization, Theorem 1.3 directly implies the equality of regular and singular types. Moreover, the existence of canonical coordinates can be seen.

Theorem 12.3. If $\partial \Omega$ is of semiregular type at $p$, then there exists a canonical coordinate for $\partial \Omega$ at $p$. Indeed, the coordinate $(z, w)$ in Lemma 12.2 (2) itself is a canonical coordinate for $\partial \Omega$ at $p$.

Proof. We will show that the coordinate $(z, w)$ in Lemma 12.2 is a canonical coordinate for $M$ at $p$. The condition (c) implies that $F(0,0)=0$ and $\nabla F(0,0)=0$. Moreover, Lemma 12.1 with the conditions (c) and (d) imply that $F$ is nondegenerate and that $\rho_{j}(F)=m_{j}$ for $j=1, \ldots, n$.

Remark 12.4. The semiregular property can be characterized by using the shapes of Newton polyhedra on canonical coordinates. Let $\partial \Omega$ be of semiregular type at $p$. It follows from Lemma 12.2 that there exists a coordinate around $p$ on which a defining function $r$ for $\Omega$ is nondegenerate and it has the Newton polyhedron of the simple form:

$$
\begin{aligned}
\mathcal{N}_{+}(r) & =\operatorname{conv}\left(\left\{m_{1} \mathbf{e}_{1}, \ldots, m_{n} \mathbf{e}_{n}, \mathbf{e}_{n+1}\right\}\right)+\mathbb{R}_{\geq}^{n} \\
& =\left\{\xi \in \mathbb{R}_{+}^{n+1}: \sum_{j=1}^{n} \frac{\xi_{j}}{m_{j}}+\xi_{n+1} \geq 1\right\} .
\end{aligned}
$$

In other words, the Newton diagram $\mathcal{N}(r)$ consists of only one facet.

Before works [12], [39], McNeal [30] and Boas and Straube [4] showed that the singular type equals the line type in the case of convex domains. Here the line type is defined by the equation (1.2) by replacing $\Gamma_{\text {reg }}$ by the set of complex lines through $p$.

For a given real hypersurface, it is not always simple matter to check the semiregularity condition and, moreover, to construct coordinates $(z, w)$ as in Lemma 12.2. J. $\mathrm{Y}$. $\mathrm{Yu}$ [38] actually constructed a coordinate as in Lemma 12.2 in the case of convex domains. 


\section{The CASE OF Reinhardt Domains}

We first recall the definition of Reinhardt domains. For $\theta=\left(\theta_{1}, \ldots, \theta_{n}\right) \in \mathbb{R}^{n}$ and $z \in \mathbb{C}^{n}$, denote

$$
e^{i \theta} \bullet z:=\left(e^{i \theta_{1}} z_{1}, \ldots, e^{i \theta_{n}} z_{n}\right)
$$

A domain $\Omega \subset \mathbb{C}^{n}$ is Reinhardt if $e^{i \theta} \bullet z \in \Omega$ whenever $z \in \Omega$ and $\theta \in \mathbb{R}^{n}$.

It has been shown by Fu, Isaev and Krantz [15] that if a hypersurface is the boundary of a pseudoconvex Reinhardt domain, then its regular and singular types agree. More strongly, we will show the existence of canonical coordinates in this case.

13.1. Invariance under the rotation. The function $F$ defined on $\mathbb{C}^{n}$ is said to be invariant under the rotation if $F$ satisfies

$$
F\left(e^{i \theta} \bullet z, \overline{e^{i \theta} \bullet z}\right)=F(z, \bar{z}) \quad \text { for all } \theta \in \mathbb{R}^{n} .
$$

Let $M$ be a real hypersurface in $\mathbb{C}^{n+1}$ and let $p$ lie on $M$.

Theorem 13.1. Let $(z, w)$ be a normalized coordinate for $M$ at $p$, on which $M$ is locally expressed by $r$ in (10.1) in Lemma 10.1. Suppose that $F$ is a nonflat plurisubharmonic function and that the principal part $F_{0}$ of $F$ is invariant under the rotation. Then the coordinate $(z, w)$ is canonical for $M$ at $p$.

Proof. Lemma 13.2, below, shows that $F_{0}$ satisfies the nondegeneracy condition. Thus, by noticing that the property (13.2) implies that $F_{0}$ contains no pure terms, Proposition 10.3 implies this theorem.

Lemma 13.2. Let $P$ be a real-valued plurisubharmonic polynomial $\not \equiv 0$. If $P$ is invariant under the rotation, then $P$ is always positive on $\left(\mathbb{C}^{*}\right)^{n}$ and, in particular, $P$ is nondegenerate.

Proof. For $\zeta \in \mathbb{C}$, let $T_{k, c}(\zeta):=\left(c_{1}, \ldots, \stackrel{(k)}{\zeta}, \ldots, c_{n}\right)$, where $k=1, \ldots, n$ and $c_{j} \in \mathbb{C}$ are fixed for $j \neq k$. Let $u: \mathbb{C} \rightarrow \mathbb{R}$ and $g: \mathbb{R}_{\geq} \rightarrow \mathbb{R}$ be the functions defined by

$$
u(\zeta, \bar{\zeta}):=P\left(T_{k, c}(\zeta), \overline{T_{k, c}(\zeta)}\right), \quad g(r):=P\left(T_{k, c}(r), \overline{T_{k, c}(r)}\right)
$$

From the hypothesis of $P$, we see

$$
g\left(r e^{i \theta}\right)=g(r) \text { for any } \theta \in \mathbb{R} \text { and } \triangle u \geq 0 \text { on } \mathbb{C} .
$$

The above properties give an inequality:

$$
\frac{d}{d r}\left(r \frac{d g}{d r}\right) \geq 0 \quad \text { for } r \geq 0
$$

By (13.3),$g$ must satisfy one of the following three conditions:

$$
g \equiv 0 \text { on } \mathbb{R}_{\geq} ; \quad g>0 \text { on } \mathbb{R}_{>} \text {and } g(0)=0 ; \quad g>0 \text { on } \mathbb{R}_{\geq} .
$$


By applying the above fact and by using an easy inductive argument on the dimension, if there exists a point $z_{0}$ in $\left(\mathbb{C}^{*}\right)^{n}$ such that $P\left(z_{0}\right)=0$, then $P \equiv 0$ on $\mathbb{C}^{n}$. This implies the assertion of the lemma.

Remark 13.3. In Lemma 13.2, "plurisubharmonic" cannot be replaced by "property PS". Consider the polynomial: $P(z, \bar{z})=\left(\left|z_{1}\right|^{2}-\left|z_{2}\right|^{2}\right)^{2}+\left|z_{1}\right|^{8}\left|z_{2}\right|^{8}$. It is easy to see that $P$ satisfies property $\mathbf{P S}$ at 0 but $P$ is degenerate.

13.2. Construction of canonical coordinates. Let us show the following theorem, which implies that the equality (1.3) holds in the pseudoconvex Reinhardt case.

Theorem 13.4. If $p$ is a boundary point of a pseudoconvex Reinhardt domain $\Omega$ with smooth boundary, then there exists a canonical coordinate for $\partial \Omega$ at $p$.

Proof. An important part of construction of a canonical coordinate has already been done in [15]. Refer for a substantial construction to [15].

Let $\Omega$ be in $\mathbb{C}^{n+1}$. Denote $\mathcal{Z}_{j}=\left\{\left(z_{1}, \ldots, z_{n+1}\right) \in \mathbb{C}^{n+1}: z_{j}=0\right\}$ for $j=1, \ldots, n+1$. Let $\mathcal{Z}=\cup_{j=1}^{n+1} \mathcal{Z}_{j}$. We define the holomorphic mapping as

$$
L^{*}\left(z_{1}, \ldots, z_{n+1}\right):=\left(\log z_{1}, \ldots, \log z_{n+1}\right),
$$

where the logarithm takes the principle branch, and $L^{*}$ is defined locally near every point of $\mathbb{C}^{n} \backslash \mathcal{Z}$.

We divide the proof into two cases.

(Generic case.) Let us first consider the case where $p \in \partial \Omega \backslash \mathcal{Z}$. As is wellknown, $L^{*}(\partial \Omega \backslash \mathcal{Z})$ is the boundary of a convex domain. This fact shows that this case is contained in [30], [4] or in the semiregular case in Section 12.

(The other case.) Let us consider the case where $p \in \partial \Omega \cap \mathcal{Z}$. It has been shown in [15] that there exists a coordinate $\left(z_{1}, \ldots, z_{n}, \zeta\right)$ near $p$ such that $p=(0, \ldots, 0,1)$, on which $\partial \Omega$ can be expressed as $\log |\zeta|+F(z, \bar{z})=0$, where $z=\left(z_{1}, \ldots, z_{n}\right)$ and $F$ satisfies all the properties in Theorem 13.1. By putting $w=\zeta-1$, the coordinate $(z, w)$ is a desired canonical coordinate around $(0,0)$. Indeed, $\partial \Omega$ can be locally expressed on $(z, w)$ near the origin as $\operatorname{Re}(w)+F(z, \bar{z})+R_{2}(w, \bar{w})=0$, where $R_{2}$ is a smooth function of $w$ satisfying $R_{2}(w)=\left(|w|^{2}\right)$. Therefore, Theorem 13.1 implies that $(z, w)$ is a canonical coordinate for $\partial \Omega$ around $p$.

Remark 13.5. In the semiregular case, the respective Newton polyhedron essentially has a very simple form (see Remark 12.4). In the Reinhardt case, the respective Newton polyhedra may take various kinds of shapes. For example, as for the real hypersurface in $\mathbb{C}^{3}$ defined by

$$
\left|z_{1}\right|^{6}+\left|z_{2}\right|^{6}+\left|z_{1} z_{2}\right|^{2}+\left|z_{3}\right|^{2}-1=0
$$

its Newton polyhedron at $(1,0,0)$ has two bounded facets. (This example is seen in [15].) 


\section{The CASE WHERE THE REGUlAR TYPE IS 4}

Let $M$ be a real hypersurface in $\mathbb{C}^{n+1}$ and let $p$ lie on $M$. Recently, McNeal and Mernik 31 and D'Angelo 9] deeply investigated order of contact in the case where $\Delta_{1}^{\mathrm{reg}}(M, p)=4$. Indeed, it was shown in [31], 9] that $\Delta_{1}(M, p)=\Delta_{1}^{\mathrm{reg}}(M, p)$ in (1.3) always holds in this case under the assumption of pseudoconvexity or property $\mathbf{P S}$ for $M$. By using the geometry of Newton polyhedra, we can see more precise geometrical structure of these hypersurfaces in the following theorem which, in particular, implies that the equality (1.3) holds.

Theorem 14.1. If a real hypersurface $M \subset \mathbb{C}^{n+1}$ satisfies property PS at $p$ and $\Delta_{1}^{\mathrm{reg}}(M, p)=4$, then there exists a canonical normalized coordinate $(z, w)$ for $M$ at $p$ on which a local defining function for $M$ can be written as in (10.1) in Lemma 10.1 with $F$ satisfying that $F$ is nondegenerate and the Newton diagram of $F$ can be expressed as

$$
\mathcal{N}(F)=\operatorname{conv}\left(\left\{4 \mathbf{e}_{1}, \ldots, 4 \mathbf{e}_{m}, 2 \mathbf{e}_{m+1}, \ldots, 2 \mathbf{e}_{n}\right\}\right),
$$

where $m$ is an integer with $1 \leq m \leq n$ (when $m=n, \mathcal{N}(F)=\operatorname{conv}\left(\left\{4 \mathbf{e}_{1}, \ldots, 4 \mathbf{e}_{n}\right\}\right)$. Furthermore, if $M$ is pseudoconvex near $p$, then its principal part $F_{0}$ of $F$ (see (11.1)) can be expressed as

$$
F_{0}(z, \bar{z})=P\left(z^{\prime}, \overline{z^{\prime}}\right)+\sum_{j=m+1}^{n}\left|z_{j}\right|^{2},
$$

$z^{\prime}=\left(z_{1}, \ldots, z_{m}\right)$ and $P$ is a nondegenerate polynomial of $z^{\prime}$ and $\overline{z^{\prime}}$ whose Newton diagram is of the form:

$$
\mathcal{N}(P)=\operatorname{conv}\left(\left\{4 \mathbf{e}_{1}, \ldots, 4 \mathbf{e}_{m}\right\}\right) .
$$

Remark 14.2. The above property (14.2) is equivalent to the following conditions:

(i) $P\left(r^{1 / 4} \bullet z^{\prime}, \overline{r^{1 / 4} \bullet z^{\prime}}\right)=r P\left(z^{\prime}, \overline{z^{\prime}}\right)$ for all $r>0$ and $z^{\prime} \in \mathbb{C}^{m}$;

(ii) $P$ is convenient, where $r^{1 / 4} \bullet z^{\prime}:=\left(r^{1 / 4} z_{1}, \ldots, r^{1 / 4} z_{m}\right)$.

Proof. Let $(z, w)$ be a normalized coordinate for $M$ with $N>4$, on which a local defining function can be expressed by using $F \in C_{0}^{\infty}\left(\mathbb{C}^{n}\right)$ as in (10.1) in Lemma 10.1. From Corollary 11.5, property PS implies that every component of coordinate of vertices of $\mathcal{N}_{+}(F)$ is even. Moreover, the condition: $\Delta_{1}^{\mathrm{reg}}(M, p)=4$ implies that the Newton diagram $\mathcal{N}(F)$ is contained in the closed half space $\left\{\xi \in \mathbb{R}^{n}:|\xi| \leq 4\right\}$. Therefore, by simple combinatorial argument and the change of the variables if necessary, $\mathcal{N}(F)$ can be essentially expressed as in a simple form:

$$
\begin{aligned}
\mathcal{N}(F) & =\operatorname{conv}\left(\left\{4 \mathbf{e}_{1}, \ldots, 4 \mathbf{e}_{l}, 2 \mathbf{e}_{l+1}, \ldots, 2 \mathbf{e}_{n}\right\}\right) \\
& =\left\{\xi \in \mathbb{R}_{\geq}^{n}: \sum_{j=1}^{l} \frac{\xi_{j}}{4}+\sum_{j=l+1}^{n} \frac{\xi_{j}}{2}=1\right\},
\end{aligned}
$$


where $l$ is some integer with $1 \leq l \leq n\left(\right.$ when $l=n, \mathcal{N}(F)=\operatorname{conv}\left(\left\{4 \mathbf{e}_{1}, \ldots, 4 \mathbf{e}_{n}\right\}\right)$.

We only consider the case when $1 \leq l \leq n-1$. (The case when $l=n$ can be considered as a particular case.)

Let $\kappa_{1}$ be the face of $\mathcal{N}_{+}(F)$ defined by $\kappa_{1}=\operatorname{conv}\left(\left\{2 \mathbf{e}_{l+1}, \ldots, 2 \mathbf{e}_{n}\right\}\right)$. Then, the $\kappa_{1}$-part of $F$ is of the form:

$$
F_{\kappa_{1}}(z, \bar{z})=\sum_{l+1 \leq j, k \leq n} C_{j k} z_{j} \bar{z}_{k}=: G(\tilde{z}, \overline{\tilde{z}})
$$

where $C_{j k} \in \mathbb{C}$ satisfy $\overline{C_{j k}}=C_{k j}$ for $l+1 \leq j, k \leq n$ and $\tilde{z}=\left(z_{l+1}, \ldots, z_{n}\right)$. There exists a unitary linear transform $U: \mathbb{C}^{n-l} \rightarrow \mathbb{C}^{n-l}$ such that

$$
G(U(\tilde{z}), \overline{U(\tilde{z})})=\sum_{j=l+1}^{n} \mu_{j}\left|z_{j}\right|^{2} \quad \text { with } \mu_{j} \in \mathbb{R} .
$$

From Proposition 11.3, property PS implies $\mu_{j} \geq 0$ for $j=l+1 \ldots, n$. Without loss of generality, there exists $m \in\{l, \ldots, n-1\}$ such that $\mu_{j}>0$ if and only if $j \in\{m+1, \ldots, n\}$. Now, we define $\hat{F} \in C_{0}^{\infty}\left(\mathbb{C}^{n}\right)$ as

$$
\hat{F}(z, \bar{z})=F\left(z_{1}, \ldots, z_{l}, U(\tilde{z}), \bar{z}_{1}, \ldots, \bar{z}_{l}, \overline{U(\tilde{z})}\right) .
$$

Since the Newton diagram $\mathcal{N}(\hat{F})$ is also contained in the half space $\left\{\xi \in \mathbb{R}^{n}:|\xi| \leq 4\right\}$, we have

$$
\mathcal{N}(\hat{F})=\operatorname{conv}\left(\left\{4 \mathbf{e}_{1}, \ldots, 4 \mathbf{e}_{m}, 2 \mathbf{e}_{m+1}, \ldots, 2 \mathbf{e}_{n}\right\}\right) .
$$

Let $\mathcal{V}$ be the set of vertices of $\mathcal{N}_{+}(\hat{F})$ defined by $\mathcal{V}=\left\{4 \mathbf{e}_{j}: j=1, \ldots, m\right\}$. Since the Newton diagram $\mathcal{N}(\hat{F})$ consists of only one facet which is regular, $\hat{F}_{\kappa}$ must be nondegenerate for every face $\kappa$ intersecting $\mathcal{V}$ from Lemma 4.5. On the other hand, every face not intersecting the set $\mathcal{V}$ is of the form

$$
\kappa_{I}=\operatorname{conv}\left(\left\{2 \mathbf{e}_{j}: j \in I\right\}\right) \quad \text { for } I \subset\{m+1, \ldots, n\} .
$$

Since the $\kappa_{I}$-part of $\hat{F}$ is of the form $\hat{F}_{\kappa_{I}}(z, \bar{z})=\sum_{j \in I} \mu_{j}\left|z_{j}\right|^{2}$ with $\mu_{j}>0$, which is nondegenerate. We have checked the nondegeneracy condition for all the faces of $\mathcal{N}_{+}(\hat{F})$ and, as a result, $\hat{F}$ is nondegenerate.

Furthermore, adding the pseudoconvex assumption, we can see that the principal part of $\hat{F}$ is of the form:

$$
\hat{F}_{0}(z, \bar{z})=P\left(z^{\prime}, \overline{z^{\prime}}\right)+\sum_{j=m+1}^{n} \mu_{j}\left|z_{j}\right|^{2},
$$

where $z^{\prime}=\left(z_{1}, \ldots, z_{m}\right)$ and $P$ satisfies the properties in the theorem. After slightly changing of scaling, we obtain an appropriate coordinate as in the theorem.

As a corollary of Theorem 14.1, we easily obtain the following. 
Corollary 14.3. If $M$ is pseudoconvex at $p$ and $\Delta_{1}^{\mathrm{reg}}(M, p)=4$, then $M$ is of semiregular type at $p$.

Proof. It follows form Theorem 14.1 that the Newton diagram $\mathcal{N}(F)$ consists of one facet. Moreover, from Lemma 11.1, the pseudoconvexity implies the plurisubharmonicities of $P$ and $F_{0}$. These give the semiregularity of $p \in M$.

Remark 14.4. It has been shown in [31] that $\Delta_{1}^{\text {reg }}(M, p)=2$ or 3 respectively implies $\Delta_{1}(M, p)=2$ or 3 without assuming properties PS (see also [24]). Even if the pseudoconvexity is assumed, the equality (1.3) can not be generalized to the case where $\Delta_{1}^{\mathrm{reg}}(M, 0)=6($ see $(1.10))$.

Remark 14.5. D. Zaitsev ([41], Theorem 2.1) has obtained a similar result to Theorem 14.1 under the pseudoconvex assumption.

Remark 14.6. McNeal and Mernik [31] gave the following interesting example: the real hypersurface $M$ in $\mathbb{C}^{3}$ defined by $r(z, w, \bar{z}, \bar{w})=\operatorname{Re}(w)+F(z, \bar{z})=0$ with

$$
F(z, \bar{z})=\left|z_{1}\right|^{2} \operatorname{Re}\left(z_{1}^{2}-z_{2}^{3}\right)+\left|z_{2}\right|^{2} \operatorname{Re}\left(z_{2}^{2}\right)-\operatorname{Re}\left(z_{1}^{2} \bar{z}_{2}\right) .
$$

This hypersurface does not satisfies property PS (in particular, pseudoconvexity) at the origin. They show that $\Delta_{1}^{\text {reg }}(M, 0)=4$ but $\Delta_{1}(M, 0)=\infty$. Let us observe this example from our point of view. Theorem 1.3 implies that there is no canonical coordinates for $M$ at the origin. The Newton diagram $\mathcal{N}(F)$ consists of the two facets:

$$
\kappa_{1}:=\operatorname{conv}(\{(4,0),(2,1)\}), \quad \kappa_{2}:=\operatorname{conv}(\{(2,1),(0,4)\}) .
$$

Notice the existence of the vertex at $(2,1)$, which is not compatible to property PS (see Corollary 11.5). The face $\kappa_{2}$ is not regular (the vector $(3,2)$ determines the face $\kappa_{1}$ ) and $F_{\kappa_{2}}(z, \bar{z})=\left|z_{2}\right|^{2} \operatorname{Re}\left(z_{2}^{2}\right)-\operatorname{Re}\left(z_{1}^{2} \bar{z}_{2}\right)$ is degenerate, which obstructs the construction of canonical coordinates.

Remark 14.7. In order to obtain the clear form (14.1) of the principal part $F_{0}$, the pseudoconvex assumption is necessary. Let us consider the real hypersurface $M$ in $\mathbb{C}^{3}$ defined by $r(z, w, \bar{z}, \bar{w})=\operatorname{Re}(w)+F(z, \bar{z})=0$ with

$$
F(z, \bar{z})=\left|z_{1}\right|^{4}+2\left|z_{1}\right|^{2} \operatorname{Re}\left(z_{2}\right)+\left|z_{2}\right|^{2} .
$$

This hypersurface satisfies property PS but is not pseudoconvex at the origin.

\section{THE CASE OF STAR-SHAPED DOMAINS}

Boas and Straube [4] not only give a simple geometrical proof of the McNeal's result [30] concerning the convex domains but also essentially generalize his result.

Theorem 15.1. (Boas-Straube [4]) Let $M$ be defined by a defining function $r$ of the form in (10.1) in Lemma 10.1. Suppose that there is a real interval $[0, \delta]$ such that for every fixed point $a=\left(a_{1}, \ldots, a_{n}\right)$ in the unit ball in $\mathbb{C}^{n}$, the function $r \mapsto F(r a, r \bar{a})$ is 
nondecreasing in $[0, \delta)$. Then the singular type is equal to the line type. In particular, the equality (1.3) holds for $M$ at the origin.

Even if $M$ satisfies the hypothesis in the above theorem, $M$ does not always admit a canonical coordinate. For example, let us consider the hypersurface defined by $r_{2}$ in Remark 1.5. Indeed, it is easy to see that this hypersurface satisfies the hypothesis in Theorem 15.1, but it does not admit any canonical coordinates.

We remark that the hypothesis in the above theorem is not necessary for the equality (1.3). Indeed, the hypothesis of Theorem 15.1 does not contain the semiregular property. The Kohn-Nirenberg example in [25, which is given by $r(z, w, \bar{z}, \bar{w})=$ $\operatorname{Re}(w)+F_{1}(z, \bar{z})$ where $F_{1}$ is as in (3.3), does not satisfies the hypothesis under any coordinate changings, but it has been seen that this hypersurface admits a canonical coordinate and the equality (1.3) holds. By using results in [25], [26], Theorem 1.3 easily provides many new examples satisfying (1.3), which are not contained in the cases discussed in Sections 12-15 (see Remark 1.5 (2)).

Acknowledgements. The author is grateful to Takeo Ohsawa, Natsuki Uehara and Yoshiki Jikumaru for many valuable comments and discussion. The author also greatly appreciates that the referee carefully and patiently read many reviced versions of this paper and gave many valuable comments which have greatly improved the readability of the paper. Furthermore, Ninh Van Thu kindly informed the author of important examples of infinite type real hypersurfaces in [13] with important suggestion, which repaired some parts of discussion in Sections 4.1 and 10.1. This work was supported by JSPS KAKENHI Grant Numbers JP15K04932, JP15H02057.

\section{REFERENCES}

[1] V. I. Arnold, S. M. Gusein-Zade and A. N. Varchenko: Singularities of differentiable maps I, II, Birkhäuser, 1985, 1988.

[2] G. Bharali and B. Stensønes: Plurisubharmonic polynomials and bumping, Math. Z. 261 (2009), 39-63.

[3] T. Bloom and I. Graham: A geometric characterization of points of type $m$ on real submanifolds of $\mathbb{C}^{n}$, J. Differetial Geometry 12 (1977), 171-182.

[4] H. P. Boas and E. J. Straube: On equality of line type and variety type of real hypersurfaces in $\mathbb{C}^{n}$. J. Geom. Anal. 2 (1992), 95-98.

[5] D. Catlin: Necessary conditions for subellipticity of the $\bar{\partial}$-Neumann problem, Ann. of Math. (2) 117 (1983), 147-171.

[6] : Subelliptic estimates for the $\bar{\partial}$-Neumann problem on pseudoconvex domains, Ann. of Math. (2) 126 (1987), 131-191.

[7] J. P. D'Angelo: Real hypersurfaces, orders of contact, and applications. Ann. of Math. (2) 115 (1982), 615-637.

[8] _ Several complex variables and the geometry of real hypersurfaces, Studies in Advanced Mathematics. CRC Press, 1993.

[9] _ : A remark on finite type conditions, J. Geom. Anal. 28 (2018), 2602-2608. 
[10] B-Y. Chen and S. Fu: The reproducing kernels and the finite type conditions, Illinois J. Math. 56 (2012), 67-83.

[11] B-Y. Chen, J. Kamimoto and T. Ohsawa: Behavior of the Bergman kernel at infinity, Math. Z. 248 (2004), 695-708.

[12] K. Diederich and G. Herbort: Pseudoconvex domains of semiregular type. Contributions to complex analysis and analytic geometry, Aspects Math., E26, 127-161, Friedr. Vieweg, Braunschweig, 1994.

[13] J. E. Fornæss and V. T. Ninh: A note on pseudoconvex hypersurfaces of infinite type in $\mathbb{C}^{n}$, To appear in Proc. Amer. Math. Soc., arXiv:1804.10087v3.

[14] J. E. Fornæss and B. Stensønes: Maximally tangent complex curves for germs of finite type $C^{\infty}$ pseudoconvex domains in $\mathbb{C}^{3}$, Math. Ann. 347 (2010), 979-991.

[15] S. Fu, A. Isaev and S. G. Krantz: Finite type conditions on Reinhardt domains, Complex Variables Theory Appl. 31 (1996), 357-363.

[16] T. Fukui: Łojasiewicz type inequalities and Newton diagrams, Proc. Amer. Math. Soc. 112 (1991), 1169-1183.

[17] R. C. Gunning: Lectures on complex analytic varieties: The local parametrization theorem, Princeton University Press, Princeton, NJ, 1970.

[18] G. Heier: Finite type and the effective Nullstellensatz, Comm. Algebra 36 (2008), 2947-2957.

[19] I. A. Ikromov and D. Müller: Fourier restriction for hypersurfaces in three dimensions and Newton polyhedra, Annals of Mathematics Studies, 194. Princeton University Press, Princeton, NJ, 2016.

[20] Y. Jikumaru: Master Thesis (Japanese), Kyushu University, 2017.

[21] J. Kamimoto: Newton polyhedra and the Bergman kernel, Math. Z. 246 (2004), 405-440.

[22] _ _ A sufficient condition for equality of regular type and singular type of real hypersurfaces, To appear in Sūrikaisekikenkyūsho Kōkyūroku.

[23] J. Kamimoto and T. Nose: Newton polyhedra and weighted oscillatory integrals with smooth phases, Trans. Amer. Math. Soc. 368 (2016), 5301-5361.

[24] J. J. Kohn: Subellipticity of the $\bar{\partial}$-Neumann problem on pseudo-convex domains: sufficient conditions, Acta Math. 142 (1979), 79-122.

[25] J. J. Kohn and L. A. Nirenberg: A pseudo-convex domain not admitting a holomorphic support function, Math. Ann. 201 (1973), 265-268.

[26] M. Kolář: Convexifiability and supporting functions in $\mathbb{C}^{2}$ : Math. Res. Lett. 2 (1995), 505-513.

[27] A. G. Kouchnirenko: Polyèdres de Newton et nombres de Milnor, Invent. Math. 32 (1976), 1-31.

[28] B. Lichtin: Estimation of Eojasiewicz exponents and Newton polygons, Invent. Math. 64 (1981), 417-429.

[29] M. Lejeune-Jalabert and B. Teissier: Clôture intégrale des idéaux et équisingularité. Ann. Fac. Sci. Toulouse Math. (6) 17 (2008), 781-859.

[30] J. McNeal: Convex domains of finite type, J. Funct. Anal. 108 (1992), 361-373.

[31] J. McNeal and L. Mernik: Regular versus singular order of contact on pseudoconvex hypersurfaces, J. Geom. Anal. 28 (2018), 2653-2669.

[32] J. McNeal and A. Némethi: The order of contact of a holomorphic ideal in $\mathbb{C}^{2}$. Math. Z. 250 (2005), 873-883.

[33] M. Oka: Non-degenerate complete intersection singularity. Actualités Mathématiques, Hermann, Paris, 1997.

[34] _ Eojasiewicz exponents of non-degenerate holomorohic and mixed functions, Kodai Math. J. 41 (2018), 620-651.

[35] G. Oleksik: The Łojasiewicz exponent of nondegenerate surface singularities, Acta Math. Hungar. 138 (2013), 179-199. 
[36] D. H. Phong and E. M. Stein: The Newton polyhedron and oscillatory integral operators, Acta Math. 179 (1997), 105-152.

[37] N. Uehara: Master Thesis (Japanese), Kyushu University, 2013.

[38] J. Y. Yu: Multitypes of convex domains, Indiana Univ. Math. J. 41 (1992), 837-849.

[39] ___ Peak functions on weakly pseudoconvex domains, Indiana Univ. Math. J. 43 (1994), 1271-1295.

[40] A. N. Varchenko: Newton polyhedra and estimation of oscillating integrals, Functional Anal. Appl., 10-3 (1976), 175-196.

[41] D. Zaitsev: A geometric approach to Catlin's boundary systems, preprint. arXiv:1704.01808,

[42] G. M. Ziegler: Lectures on Polytopes, Graduate Texts in Mathematics, 152. Springer-Verlag, New York, 1995.

Faculty of Mathematics, Kyushu University, Motooka 744, Nishi-Ku, Fukuoka, 8190395, JAPAN

E-mail address: joe@math.kyushu-u.ac.jp 